\title{
A family of non-oscillatory 6-point interpolatory subdivision schemes
}

\section{Rosa Donat, Sergio López-Ureña \& Maria Santágueda}

Advances in Computational Mathematics

Modelling in Science and Engineering

ISSN 1019-7168

Adv Comput Math

DOI 10.1007/s10444-016-9509-5

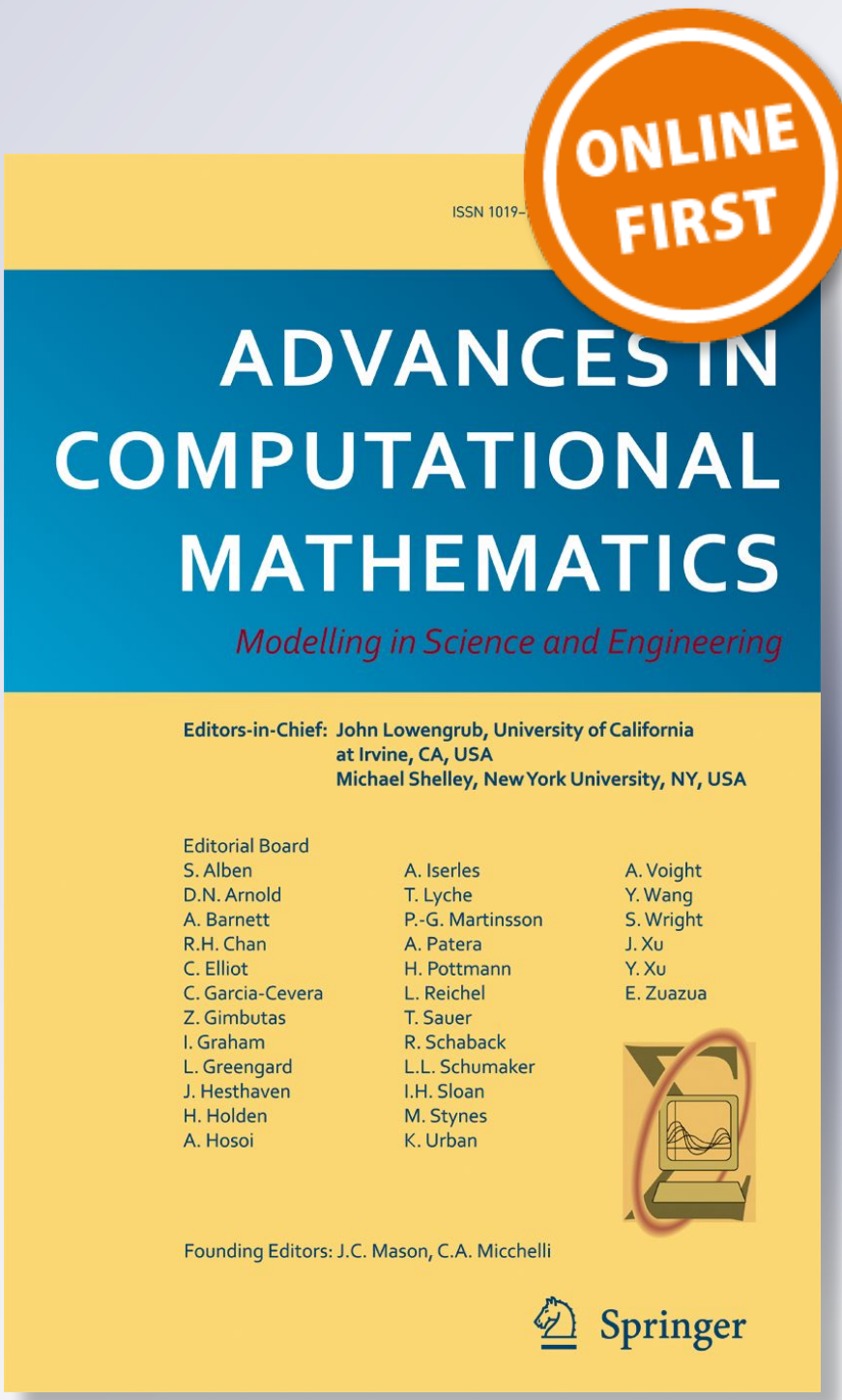

Springer 
Your article is protected by copyright and all rights are held exclusively by Springer Science +Business Media New York. This e-offprint is for personal use only and shall not be selfarchived in electronic repositories. If you wish to self-archive your article, please use the accepted manuscript version for posting on your own website. You may further deposit the accepted manuscript version in any repository, provided it is only made publicly available 12 months after official publication or later and provided acknowledgement is given to the original source of publication and a link is inserted to the published article on Springer's website. The link must be accompanied by the following text: "The final publication is available at link.springer.com". 


\title{
A family of non-oscillatory 6-point interpolatory subdivision schemes
}

\author{
Rosa Donat ${ }^{1}$ - Sergio López-Ureña ${ }^{1}$. \\ Maria Santágueda ${ }^{2}$
}

\begin{abstract}
In this paper we propose and analyze a new family of nonlinear subdivision schemes which can be considered non-oscillatory versions of the 6-point Deslauries-Dubuc (DD) interpolatory scheme, just as the Power $_{p}$ schemes are considered nonlinear non-oscillatory versions of the 4-point DD interpolatory scheme. Their design principle may be related to that of the Power $_{p}$ schemes and it is based on a weighted analog of the Power $p$ mean. We prove that the new schemes reproduce exactly polynomials of degree three and stay 'close' to the 6-point DD scheme in smooth regions. In addition, we prove that the first and second difference schemes are well defined for each member of the family, which allows us to give a simple proof of the uniform convergence of these schemes and also to study their stability as in $[19,22]$. However our theoretical study of stability is not conclusive and we perform a series of numerical experiments that seem to point out that only a few members of the new family of schemes are stable. On the other hand, extensive numerical testing reveals that, for smooth data, the approximation order and the regularity of the limit function may be similar to that of the 6-point DD scheme and larger than what is obtained with the $\operatorname{Power}_{p}$ schemes.
\end{abstract}

Communicated by: Yuesheng Xu

Rosa Donat

donat@uv.es

Sergio López-Ureña

sergio.lopez-urena@uv.es

Maria Santágueda

santague@edu.uji.es

1 Departament de Matemàtiques, Universitat de València, Doctor Moliner Street 50, 46100

Burjassot, Valencia, Spain

2 Departament d'Educació, Universitat Jaume I de Castelló de la Plana, Castelló de la Plana, Spain 
Keywords Nonlinear subdivision schemes · Convergence · Stability · Approximation order $\cdot$ Non-oscillatory

\section{Introduction}

Subdivision schemes are recursive processes used for the fast generation of curves and surfaces in computer-aided geometric design, as well as an essential ingredient in many multiscale algorithms used in data compression. In some applications, the given data need to be retained at each step of the refinement process, which requires the use of interpolatory subdivision schemes. The so-called Deslauries-Dubuc (DD henceforth) subdivision schemes [6] are a well known family of interpolatory subdivision schemes which can be interpreted as a recursive application of a piecewise polynomial interpolatory tool $[10,11]$. A general setting by which a piecewise polynomial interpolation technique can be used to provide the set of local rules that defines a subdivision scheme has been described in [10]: Assuming that $\chi^{l} \subset \chi^{l+1}$ are two nested grids on $\mathbb{R}^{m}, f^{l}$ is a set of known data associated to the grid $\chi^{l}$ and $\mathcal{I}[x, \cdot]$ is a piecewise polynomial reconstruction technique, new data associated to the grid $\chi^{l+1}$ can be generated as follows

$$
f_{i}^{l+1}=\mathcal{I}\left[x_{i}^{l+1}, f^{l}\right], \quad \text { for } x_{i}^{l+1} \in \chi^{l+1} .
$$

This process allows to define a recursive subdivision scheme where sequences of values on denser and denser meshes are obtained according to the set of local rules derived from $\mathcal{I}[x, \cdot]$. Clearly (1) leads to an interpolatory subdivision scheme if $\mathcal{I}$ is an interpolatory reconstruction, i.e. $\mathcal{I}\left[x_{i}^{l}, f^{l}\right]=f_{i}^{l}, \forall x_{i}^{l} \in \chi^{l}$. For $m=1$ and a binary refinement strategy, i.e. $x_{2 i}^{l+1}=x_{i}^{l}$ and $\chi^{l+1} \backslash \chi^{l} \equiv\left\{x_{2 j+1}^{l+1}\right\}_{j \in \mathbb{Z}}$, we have

$$
\begin{aligned}
f_{2 i}^{l+1} & =\mathcal{I}\left[x_{2 i}^{l+1}, f^{l}\right]=\mathcal{I}\left[x_{i}^{l}, f^{l}\right]=f_{i}^{l} \\
f_{2 i+1}^{l+1} & =\mathcal{I}\left[x_{2 i+1}^{l+1}, f^{l}\right]
\end{aligned}
$$

so that values on a given mesh are 'copied' at the same location on higher resolution levels, while the interpolatory technique $\mathcal{I}[\cdot, \cdot]$ specifies the local rules used for the generation of new data values.

It is well known (see e.g. $[9,10])$ that the DD subdivision schemes can be written in the form (2) with $\mathcal{I}(\cdot, \cdot)$ a Lagrange interpolatory reconstruction that considers an interpolatory stencil centered around the evaluation point. In general, the use of piecewise polynomial Lagrange interpolation based on a stencil that uses $l$ points to the left and $r$ points to the right of the evaluation point leads to a linear (i.e. data-independent) subdivision scheme that can be written as

$$
\left(S_{l, r} f\right)_{2 i}=f_{i}, \quad\left(S_{l, r} f\right)_{2 i+1}=\psi_{l, r}\left(f_{i-l}, \ldots, f_{i+r-1}\right)=\sum_{j=-l}^{r-1} a_{j}^{l, r} f_{i+j}, \quad f \in l_{\infty}(\mathbb{Z})
$$



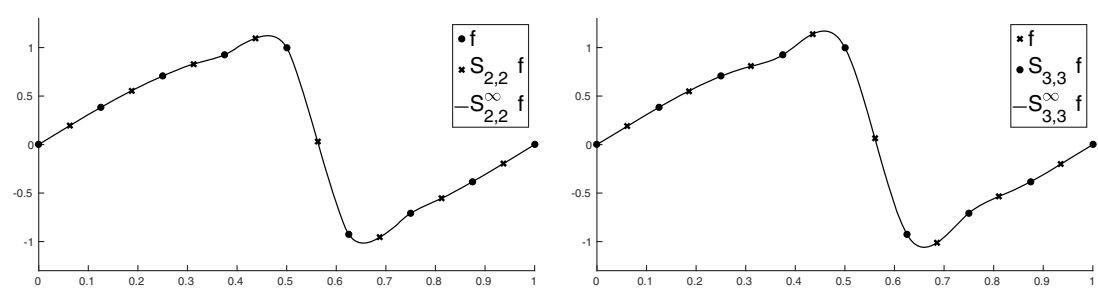

Fig. 1 Oscillatory behavior of DD schemes. For each scheme $S, S^{\infty} f$ is the corresponding limit function

with mask coefficients $a_{j}^{l, r}$ that can be computed from the interpolatory rule $\mathcal{I}[\cdot, \cdot \cdot]$ (see $[9,10])$. It is well known that these schemes lead to a Gibbs-like oscillatory behavior when applied to discrete data with large gradients (see Fig. 1) and several nonlinear piecewise polynomial interpolatory techniques have been considered in the literature, within this same framework, in an attempt to construct interpolatory subdivision schemes that avoid undesired oscillations. Examples of such schemes are the ENO-WENO subdivision schemes [10, 11, 14, 16] or the PPH scheme [2, 4].

The oscillatory behavior displayed in Fig. 1 is typical of data-independent subdivision schemes based on Lagrange interpolation, which does not preserve the shape properties of data with large gradients when the degree of the polynomial pieces is larger than 1. ENO-WENO subdivision schemes manage to avoid the Gibbslike oscillatory behavior by selecting an appropriate stencil for the interpolatory reconstruction $[10,11,14,16]$. Other nonlinear interpolatory subdivision schemes, like the Power $_{p}$ schemes [17] or the shape-preserving schemes in [8], owe their non-oscillatory character to the judicious use of certain nonlinear averages.

The aim of this paper is to design, and analyze, non-oscillatory 6-point schemes that can be considered nonlinear analogs of the 6-point DD linear scheme, $S_{3,3}$, in the same sense as the Power $p$ schemes are considered nonlinear, non-oscillatory versions of $S_{2,2}$, the 4 point DD subdivision scheme. For this, we shall use a weighted nonlinear average defined in [23] which generalizes the Power ${ }_{p}$ mean defined in [15] and used in the design of the $\operatorname{Power}_{p}$ schemes [17]. The new schemes proposed in this paper can be written in the following general form

$$
\left(S_{\mathcal{N}} f\right)_{n}=\left(S_{\mathcal{L}} f\right)_{n}+\mathcal{F}(\delta f)_{n}, \quad \forall n \in \mathbb{Z}, \quad \forall f \in l^{\infty}(\mathbb{Z}),
$$

where $\mathcal{F}: l^{\infty}(\mathbb{Z}) \rightarrow l^{\infty}(\mathbb{Z})$ is a nonlinear operator, $\delta: l^{\infty}(\mathbb{Z}) \rightarrow l^{\infty}(\mathbb{Z})$ is linear and continuous and $S_{\mathcal{L}}$ is a linear and convergent subdivision scheme. The Power $p$ schemes and other related subdivision schemes considered in [1, 3, 22] can also be written in the form (4), which allowed the authors to study their convergence and stability by using the following results [1].

Theorem 1 Let $S_{\mathcal{N}}$ be a nonlinear subdivision scheme which can be written in the form (4). 
The scheme $S_{\mathcal{N}}$ is uniformly convergent provided that $\mathcal{F}$ and $\delta$ satisfy the following conditions:

$$
\begin{array}{lll}
\text { C1. } \exists M>0: & \|\mathcal{F}(f)\|_{\infty} \leq M\|f\|_{\infty} & \forall f \in l^{\infty}(\mathbb{Z}) \\
\text { C2. } \exists L>0,0<T<1:\left\|\delta S_{\mathcal{N}}^{L}(f)\right\|_{\infty} \leq T\|\delta f\|_{\infty} & \forall f \in l^{\infty}(\mathbb{Z})
\end{array}
$$

The scheme $S_{\mathcal{N}}$ is (Lipschitz) stable, i.e. $\exists C>0$ such that

$$
\left\|S_{\mathcal{N}}^{j} f-S_{\mathcal{N}}^{j} g\right\|_{\infty} \leq C\|f-g\|_{\infty} \quad \forall f, g \in l^{\infty}(\mathbb{Z}), \quad \forall j \geq 0,
$$

provided that $\mathcal{F}$ and $\delta$ satisfy the following conditions:

$$
\begin{array}{ll}
\text { S1. } \exists M>0: & \|\mathcal{F}(f)-\mathcal{F}(g)\|_{\infty} \leq M\|f-g\|_{\infty}, \quad \forall f, g \in l^{\infty}(\mathbb{Z}) \\
\text { S2. } \exists L>0,0<T<1: & \left\|\delta\left(S_{\mathcal{N}}^{L}(f)-S_{\mathcal{N}}^{L}(g)\right)\right\|_{\infty} \leq T\|\delta(f-g)\|_{\infty}, \quad \forall f, g \in l^{\infty}(\mathbb{Z})
\end{array}
$$

Remark 2 If a scheme of the form (4) is convergent, the smoothness of the limit functions $S_{\mathcal{N}}^{\infty} f, f \in l_{\infty}(\mathbb{Z})$, may be related to the smoothness of $S_{\mathcal{L}}^{\infty} f$. In particular it can be shown (see $[12,17]$ ) that if $S_{\mathcal{L}}$ is $C^{r-}$ convergent $^{1}$ then $S_{\mathcal{N}}$ is at least $C^{s-}$ convergent with $s=\min \left\{-\frac{\log _{2}(T)}{L}, r\right\}$.

The new schemes proposed in this paper share other features with the $\operatorname{Power}_{p}$ schemes. For both families of schemes the linear operator in Eq. 4 can be considered as $\delta=\nabla^{2}$, where

$(\nabla f)_{n}=f_{n+1}-f_{n}$,

$\left(\nabla^{m+1} f\right)_{n}=\left(\nabla^{m} f\right)_{n+1}-\left(\nabla^{m} f\right)_{n}, \quad m \geq 1, \quad n \in \mathbb{Z}$,

and the subdivision schemes are defined by piecewise smooth functions that are globally Lipschitz.

The paper is organized as follows: in Section 2 we provide an explanation of the non-oscillatory character of the Power $p$ schemes which can be used as a design tool to obtain new families of non-oscillatory 6-point interpolatory subdivision schemes. These shall require a nonlinear analog of the Power $_{p}$ mean, the Weighted Power $p$, proposed in [23]. The new families of 6-point schemes are defined and analyzed in Section 3. In this section we examine the polynomial reproduction properties and the existence of difference schemes, as well as the convergence and approximation properties of the new families of schemes.

Section 4 is devoted to the issue of the stability of the new schemes. In Section 4.1 we study the Weighted Power $_{p}$ mean, and its Generalized Gradient, an essential ingredient in the application of the theory developed in [19] for the study of the stability of a nonlinear scheme. The limitations of this theory in the study of the stability of the proposed schemes are analyzed in Section 4.2. In Section 5 we study the stability issue from a computational point of view, and also present several numerical examples that illustrate our theoretical results. We close in Section 6 with some conclusions.

\footnotetext{
${ }^{1}$ For $0<r \leq 1, C^{r-}$ is the space of bounded continuous functions satisfying $|F(x)-F(y)| \leq C|x-y|^{r_{1}}$, $\forall r_{1}<r, \forall x, y \in \mathbb{R},|x-y|<1$, with $C>0$ independent of $x, y$. For $r>1, r=p+\beta, p \in \mathbb{N}$, $0<\beta \leq 1$, it is required that $F^{(p)} \in C^{\beta-}$.
} 
In addition, the relation between the Generalized Gradients of the piecewise smooth functions that define a nonlinear scheme, and the contractivity of such scheme is carefully explained in an Appendix to this paper.

\section{Nonlinear averages and Non-oscillatory schemes}

The Power $_{p}$ interpolatory subdivision schemes [17, 19] are binary interpolatory subdivision schemes for which the generation of new data values (at odd points) is given by the following rule

$$
\left(S_{\mathrm{H}_{p}} f\right)_{2 n+1}=\psi_{\mathrm{H}_{p}}\left(f_{n-1}, f_{n}, f_{n+1}, f_{n+2}\right)=\frac{1}{2}\left(f_{n}+f_{n+1}\right)-\frac{1}{8} \mathrm{H}_{p}\left(\nabla^{2} f_{n-1}, \nabla^{2} f_{n}\right)
$$

where

$$
\mathrm{H}_{p}(x, y)=\frac{\operatorname{sgn}(x)+\operatorname{sgn}(y)}{2} \frac{|x+y|}{2}\left(1-\left|\frac{x-y}{x+y}\right|^{p}\right)
$$

is the so-called Power $p$ mean [15], a nonlinear function that satisfies (see [15, 17] for details)

(a) $\mathrm{H}_{p}(x, x)=x, \quad$ (b) $\min \{|x|,|y|\} \leq\left|\mathrm{H}_{p}(x, y)\right| \leq \min \{\max (|x|,|y|), p \min (|x|,|y|)\}$.

It is straightforward to see that $\psi_{2,2}$ in Eq. 3 can be written as

$$
\begin{aligned}
& \psi_{2,2}\left(f_{n-1}, f_{n}, f_{n+1}, f_{n+2}\right)=\frac{1}{2}\left(f_{n}+f_{n+1}\right) \\
& \quad-\frac{1}{8} \operatorname{ave~}_{\frac{1}{2}, \frac{1}{2}}\left(\nabla^{2} f_{n-1}, \nabla^{2} f_{n}\right), \text { ave }_{\frac{1}{2}, \frac{1}{2}}(x, y)=\frac{1}{2} x+\frac{1}{2} y .
\end{aligned}
$$

The obvious similarity between Eq. 9 and Eq. 6 makes the Power $_{p}$ schemes nonlinear versions of the 4-point DD scheme. In addition, if $f=\left(f_{i}\right)_{i \in \mathbb{Z}}, f_{i}=F\left(x_{i}\right)$ with $F(x)$ a smooth convex function, and $\mathcal{X}=\left\{x_{i}\right\}$ an $h$-uniform grid, it can be proven that

$$
\left\|S_{2,2} f-S_{\mathrm{H}_{p}} f\right\|_{\infty}=\mathcal{O}\left(h^{p+2}\right) .
$$

This property is obtained from the following relation (see e.g. [17]), which holds for $x \cdot y>0, p \geq 1$

$$
\left|\operatorname{ave}_{\frac{1}{2}, \frac{1}{2}}(x, y)-\mathrm{H}_{p}(x, y)\right|=\frac{1}{2} \frac{|x-y|^{p}}{|x+y|^{p-1}} .
$$

On the other hand, as shown in Fig. 2, the behavior of $S_{\mathrm{H}_{p}}$ when refining discrete data with large gradients is quite different from that of $S_{2,2}$. In what follows, we give an explanation of the lack of oscillations observed in Fig. 2 which may be used to design 6-point nonlinear analogs of $S_{3,3}$, the 6 point DD scheme. The starting point 

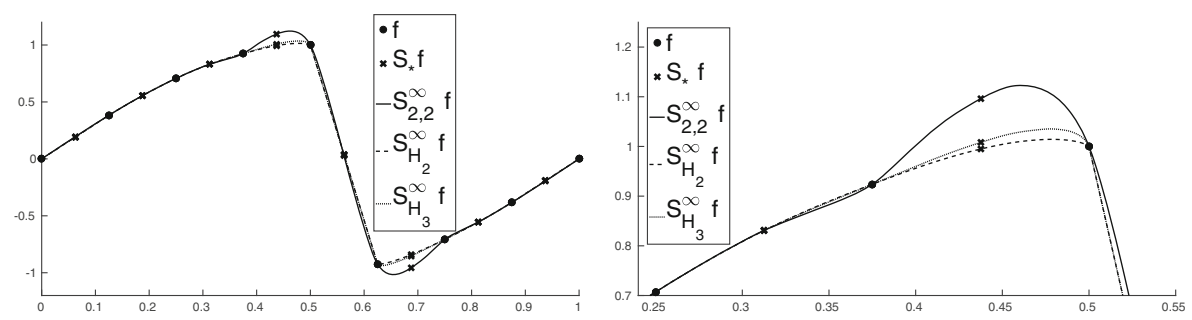

Fig. 2 Non-oscillatory behavior of Power $_{p}$ schemes, $S_{H_{p}}$, for $p=2$, 3, compared to the 4-point DD scheme, $S_{2,2}$

in our construction is the following relation,

$$
S_{l, r}=\frac{r-1 / 2}{l+r-1} S_{l, r-1}+\frac{l-1 / 2}{l+r-1} S_{l-1, r}, \quad l, r \geq 1
$$

which follows from Neville's Algorithm for Lagrange interpolation (see e.g. [13]). Moreover, it is not difficult to see that we can write, for $l+r>1$

$$
S_{l, r}=S_{1,1}+\mathcal{L}_{l, r} \circ \nabla^{2},
$$

where $\mathcal{L}_{l, r}$ is a linear operator such that $\left(\mathcal{L}_{l, r} f\right)_{2 n}=0$, due to the interpolatory property. For the two schemes below

$$
\left(S_{2,1} f\right)_{2 n+1}=\left(S_{1,1} f\right)_{2 n+1}-\frac{1}{8} \nabla^{2} f_{n-1}, \quad\left(S_{1,2} f\right)_{2 n+1}=\left(S_{1,1} f\right)_{2 n+1}-\frac{1}{8} \nabla^{2} f_{n,}
$$

hence

$$
\left(\mathcal{L}_{2,1} f\right)_{2 n+1}=-\frac{1}{8} f_{n-1} \quad\left(\mathcal{L}_{1,2} f\right)_{2 n+1}=-\frac{1}{8} f_{n} .
$$

From Eq. 12, for $l=2, r=2$, we get

$$
S_{2,2}=\frac{1}{2} S_{2,1}+\frac{1}{2} S_{1,2}=S_{1,1}+\operatorname{ave}_{\frac{1}{2}, \frac{1}{2}}\left(\mathcal{L}_{1,2} \circ \nabla^{2}, \mathcal{L}_{2,1} \circ \nabla^{2}\right)
$$

while for $S_{\mathrm{H}_{p}}$ in Eq. 6 we can write

$$
S_{\mathrm{H}_{p}}=S_{1,1}+\mathrm{H}_{p}\left(\mathcal{L}_{1,2} \circ \nabla^{2}, \mathcal{L}_{2,1} \circ \nabla^{2}\right) .
$$

Taking into account (15) and Eq. 16, the behavior of the $S_{2,2}$ and $S_{\mathrm{H}_{p}}$ schemes may be explained in terms of the interpolatory stencils associated to the schemes $S_{2,1}$ and $S_{1,2}$, shown in Fig. 4.

It is a well known fact that any Lagrange-type interpolatory technique suffers an $\mathcal{O}(1)$ accuracy loss as soon as the interpolatory stencil crosses a discontinuity. The data in Figs. 1-2 correspond to $f_{i}=F\left(x_{i}\right), i \in \mathbb{Z}, F(x)$ smooth except for an isolated discontinuity $\theta \in\left(x_{m}, x_{m+1}\right)$. For these data

$$
\nabla^{2} f_{j}=\mathcal{O}\left(h^{2}\right), \quad j \neq m-1, m, \quad \nabla^{2} f_{m-1}=\mathcal{O}(1)=\nabla^{2} f_{m} .
$$

Since

$$
\operatorname{ave}_{\frac{1}{2}, \frac{1}{2}}\left(\mathcal{O}\left(h^{r}\right), \mathcal{O}\left(h^{s}\right)\right)=\mathcal{O}\left(h^{\min (r, s)}\right), \quad r>0, s>0, \quad 0<h<1,
$$



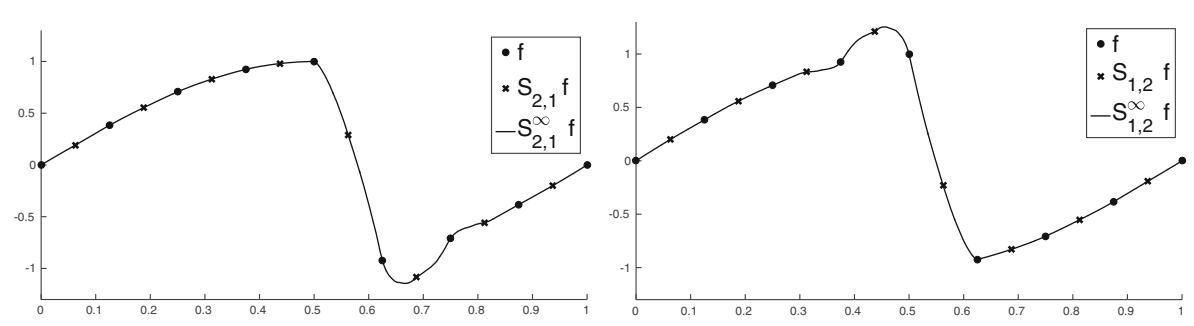

Fig. 3 Gibbs-like oscillatory behavior of $S_{1,2}, S_{2,1}$ schemes

we get (see Fig. 4)

$$
\begin{array}{ll}
\left(S_{2,2} f\right)_{2 j+1}=\left(S_{1,1} f\right)_{2 j+1}+\mathcal{O}\left(h^{2}\right) & j \notin\{m-1, m, m+1\}, \\
\left(S_{2,2} f\right)_{2 j+1}=\left(S_{1,1} f\right)_{2 j+1}+\mathcal{O}(1) & j \in\{m-1, m, m+1\} .
\end{array}
$$

The values $\left(S_{2,2} f\right)_{2 j+1}$ are displayed as $\times$ in Figs. 1-2, and the $\mathcal{O}(1)$ perturbations in Eq. 19 are clearly visible in Fig. 2 at the intervals adjacent to the one containing the discontinuity. After repeated application of the subdivision scheme, they cause the oscillations observed in the limit function, $S_{2,2}^{\infty} f$.

On the other hand, $\operatorname{since} \min \{|x|,|y|\} \leq\left|H_{p}(x, y)\right| \leq p \min \{|x|,|y|\}$ for $x y>0$, we may write

$$
\left(S_{\mathrm{H}_{p}} f\right)_{2 j+1} \approx\left(S_{1,1} f\right)_{2 j+1}+\left\{\begin{array}{ll}
\left(\mathcal{L}_{2,1} \circ \nabla^{2} f\right)_{2 n+1} & j=m-1 \\
\left(\mathcal{L}_{1,2} \circ \nabla^{2} f\right)_{2 n+1} & j=m+1
\end{array}= \begin{cases}\left(S_{2,1} f\right)_{2 j+1} & j=m-1 \\
\left(S_{1,2} f\right)_{2 j+1} & j=m+1\end{cases}\right.
$$

Thus, the behavior of the $S_{\mathrm{H}_{p}}$ schemes at the intervals neighboring the singularity is closer to the behavior of the $S_{l, r}$ scheme, $(l, r)=\{(1,2),(2,1)\}$, which uses a stencil that does not cross the singularity, see Figs. 2, 3 and 4, which is, ultimately, the reason for the lack of oscillatory behavior.

We would like to proceed in a similar manner in order to limit the influence of schemes with singularity-crossing stencils for the 6-point DD linear scheme. For $S_{3,3}$ we may write

$$
S_{3,3}=\frac{1}{2} S_{2,3}+\frac{1}{2} S_{3,2}=\frac{1}{2}\left(\frac{3}{8} S_{3,1}+\frac{5}{8} S_{2,2}\right)+\frac{1}{2}\left(\frac{3}{8} S_{1,3}+\frac{5}{8} S_{2,2}\right) .
$$

The stencils associated to the schemes $S_{2,3}$ and $S_{3,2}$ would not allow to avoid oscillations at all intervals neighboring a singularity in the data. On the other hand,

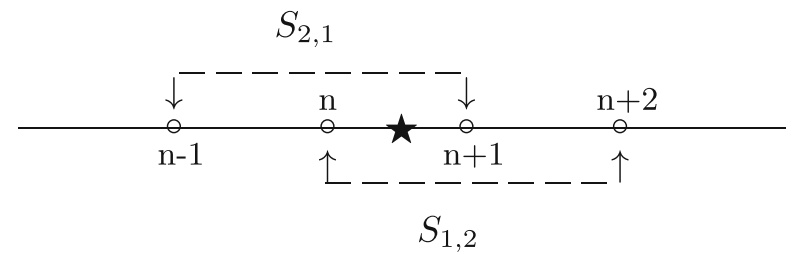

Fig. $4 \star$ evaluation point, o points in $\mathcal{S}_{2,2}$-stencil. Discontinuous lines: stencils for $\mathcal{S}_{1,2}, \mathcal{S}_{2,1}$ 


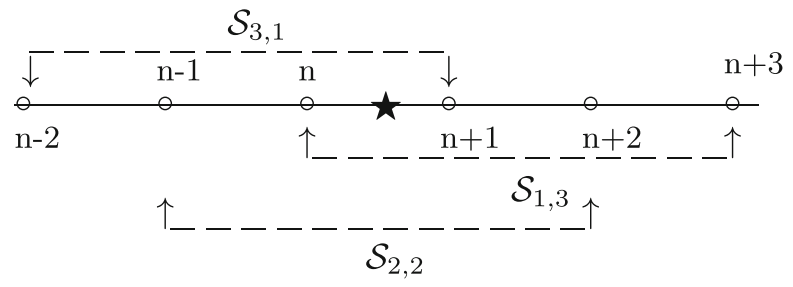

Fig. $5 \star$ : evaluation point, o points in $\mathcal{S}_{3,3}$-stencil. Discontinuous lines: stencils for $\mathcal{S}_{2,2}, \mathcal{S}_{1,3}$ y $\mathcal{S}_{3,1}$

given the distribution of the stencils for $S_{3,1}, S_{1,3}, S_{2,2}$ shown in Fig. 5, we conclude that it might be possible to design non-oscillatory versions of the $S_{3,3}$ scheme by considering nonlinear analogs of the linear averages involved in Eq. 20 that allow us to remain closer to the $S_{l, r}$ scheme whose interpolatory stencil does not cross the singularity. For the general weighted average expression

$$
\operatorname{ave}_{a, b}(x, y):=a x+b y, \quad 0 \leq a, b \leq 1, \quad a+b=1,
$$

we may consider the Weighted Power $_{p}$ mean proposed in [23].

Definition 3 Weighted-Power $_{p}$ mean [23]. Let be $a>0, b>0$ satisfying $a+b=1$, and $p \geq 1$. Then, $\forall x, y \in \mathbb{R}$.

$$
\mathrm{W}_{p, a, b}(x, y):=\operatorname{sgn}(x, y)|a x+b y|\left(1-\frac{|x-y|^{p}}{\left(M+\frac{m}{\alpha}\right)(M+\alpha m)^{p-1}}\right),
$$

where $M=\max \{|x|,|y|\}, m=\min \{|x|,|y|\}, \alpha=\max \{a, b\} / \min \{a, b\}$, $\operatorname{sgn}(x, y)=\frac{1}{2}(\operatorname{sign}(x)+\operatorname{sign}(y))$.

It is proven in [23] that $\mathrm{W}_{p, a, b}(x, y)$ generalizes the $\mathrm{H}_{p}$ mean. Indeed, it can be easily checked that

$$
\mathrm{W}_{p, a, b}(x, x)=x, \quad \mathrm{~W}_{p, a, b}(x, y)=\mathrm{W}_{p, b, a}(y, x), \quad \mathrm{W}_{p, \frac{1}{2}, \frac{1}{2}}(x, y)=\mathrm{H}_{p}(x, y) .
$$

We recall next some of the properties of $\mathrm{W}_{p, a, b}(x, y)$ in Eq. 22. The reader is referred to [23] for the proofs.

Proposition 4 The function $W_{p, a, b}(x, y)$ in (22) satisfies the following properties.

$$
\text { (a) }\left|W_{p, a, b}(x, y)\right| \leq|a x+b y| \quad \text { (b) } \frac{1}{\alpha} \min \{|x|,|y|\} \leq\left|W_{p, a, b}(x, y)\right| \leq p \alpha \min \{|x|,|y|\} \text {. }
$$




\section{6-point Nonlinear, Non-oscillatory schemes}

Taking into account Eqs. 20 and 13, we can write

$$
\begin{aligned}
& S_{3,3}=S_{1,1}+\operatorname{ave}_{\frac{3}{8}, \frac{5}{8}}\left(\operatorname{ave}_{\frac{1}{2}, \frac{1}{2}}\left(\mathcal{L}_{1,3} \circ \nabla^{2}, \mathcal{L}_{3,1} \circ \nabla^{2}\right), \mathcal{L}_{2,2} \circ \nabla^{2}\right), \\
& S_{3,3}=S_{1,1}+\operatorname{ave}_{\frac{1}{2}, \frac{1}{2}}\left(\operatorname{ave}_{\frac{3}{8}, \frac{5}{8}}\left(\mathcal{L}_{1,3} \circ \nabla^{2}, \mathcal{L}_{2,2} \circ \nabla^{2}\right), \operatorname{ave}_{\frac{3}{8}, \frac{5}{8}}\left(\mathcal{L}_{3,1} \circ \nabla^{2}, \mathcal{L}_{2,2} \circ \nabla^{2}\right) .\right.
\end{aligned}
$$

where it can easily be shown that

$$
\begin{aligned}
& \left(\mathcal{L}_{3,1} f\right)_{2 n+1}=-\frac{1}{16}\left(-f_{n-2}+3 f_{n-1}\right), \quad\left(\mathcal{L}_{1,3} f\right)_{2 n+1}=-\frac{1}{16}\left(3 f_{n}-f_{n+1}\right), \\
& \left(\mathcal{L}_{2,2} f\right)_{2 n+1}=-\frac{1}{16}\left(f_{n-1}+f_{n}\right) .
\end{aligned}
$$

We may obtain two families of nonlinear 6-point schemes simply by replacing each linear average by the appropriate nonlinear mean (recall that $\mathrm{W}_{p, \frac{1}{2}, \frac{1}{2}}=\mathrm{H}_{p}$ ).

$$
\begin{aligned}
& \operatorname{SWH}_{p, q}=S_{1,1}+\mathrm{W}_{p, \frac{3}{8}, \frac{5}{8}}\left(\mathrm{H}_{q}\left(\mathcal{L}_{1,3} \circ \nabla^{2}, \mathcal{L}_{3,1} \circ \nabla^{2}\right), \mathcal{L}_{2,2} \circ \nabla^{2}\right), \\
& \operatorname{SHW}_{q, p}=S_{1,1}+\mathrm{H}_{q}\left(\mathrm{~W}_{p, \frac{3}{8}, \frac{5}{8}}\left(\mathcal{L}_{1,3} \circ \nabla^{2}, \mathcal{L}_{2,2} \circ \nabla^{2}\right), \mathrm{W}_{p, \frac{3}{8}, \frac{5}{8}}\left(\mathcal{L}_{3,1} \circ \nabla^{2}, \mathcal{L}_{2,2} \circ \nabla^{2}\right) .\right.
\end{aligned}
$$

Because of Eq. 24-(b), these schemes remain closer to the subdivision scheme with the least oscillatory behavior, hence they are expected to display a non-oscillatory behavior similar to that of the Power $_{p}$ schemes, see Fig. 6 .

In addition, since these subdivision schemes can be written as a nonlinear perturbation of the monotone $S_{1,1}$ linear scheme, many of their properties can be analyzed with the same tools used in [19] for the Power ${ }_{p}$ schemes. We examine next the polynomial reproduction properties of these families of nonlinear subdivision schemes and the existence of difference schemes.
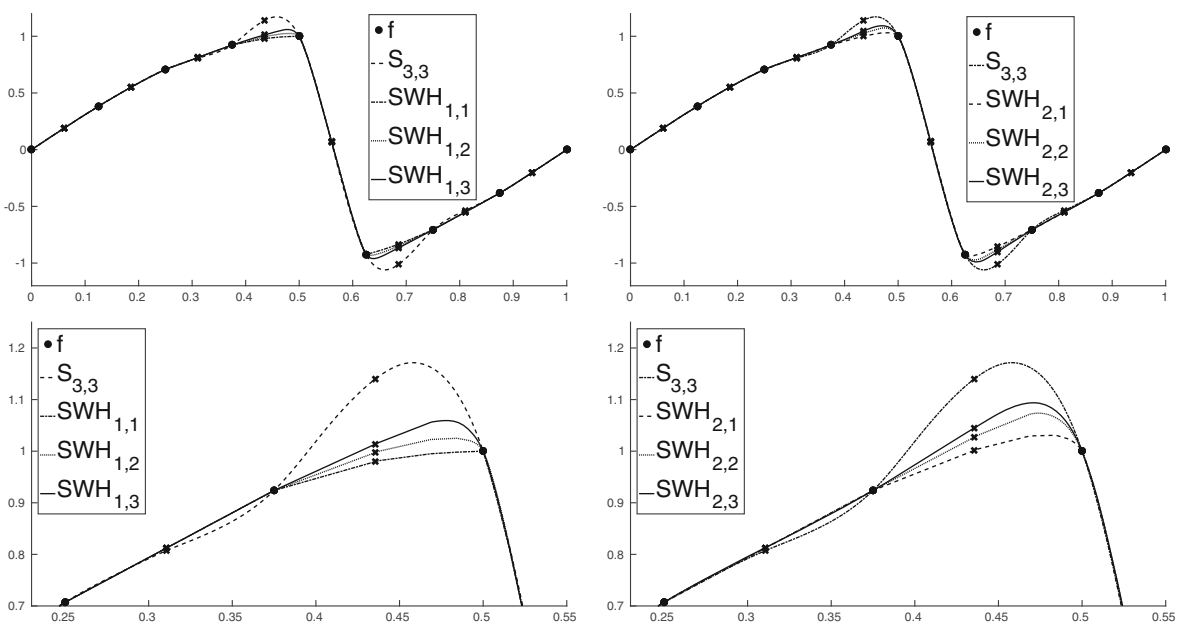

Fig. 6 Non-oscillatory behavior of $\mathrm{SWH}_{p, q}$. For each scheme $S$ : Crosses denote $S f$. Lines denote $S^{\infty} f$ 


\subsection{Polynomial reproduction properties and difference schemes}

Throughout this section, we denote by $\Pi_{k}$ the set of polynomials of degree $\leq k$, and by $\mathbf{1}$ the constant sequence given by $\mathbf{1}_{i}=1, i \in \mathbb{Z}$.

Proposition 5 The schemes $S W H_{p, q}, S H W_{q, p}$ reproduce exactly $\Pi_{3}$.

Proof Since $S_{3,1}, S_{2,2}$ and $S_{1,3}$ reproduce $\Pi_{3}$ exactly, we have that for $P \in \Pi_{3}$ and $f=\left.P\right|_{\mathbb{Z}}$

$$
\left(S_{3,1} f\right)_{2 n+1}=\left(S_{2,2} f\right)_{2 n+1}=\left(S_{1,3} f\right)_{2 n+1}=P(n+1 / 2), \quad \forall n \in \mathbb{Z}
$$

and, from Eq. 13, $\left(\mathcal{L}_{3,1}\left(\nabla^{2} f\right)\right)_{2 n+1}=\left(\mathcal{L}_{2,2}\left(\nabla^{2} f\right)\right)_{2 n+1}=\left(\mathcal{L}_{1,3}\left(\nabla^{2} f\right)\right)_{2 n+1}$. Since $\mathrm{W}_{p, a, b}(x, x)=x, \forall p, q \geq 1$

$$
\begin{aligned}
\left(\mathrm{SWH}_{p, q} f\right)_{2 n+1} & =\left(\mathrm{SHW}_{q, p} f\right)_{2 n+1}=\left(S_{1,1} f\right)_{2 n+1}-\left(\mathcal{L}_{2,2}\left(\nabla^{2} f\right)\right)_{2 n+1} \\
& =\left(S_{2,2} f\right)_{2 n+1}=P(n+1 / 2) .
\end{aligned}
$$

In the linear case, the relation between exact polynomial reproduction and the existence of the associated difference schemes $S^{[l]}$ with $S^{[0]}=S$, and $\nabla^{l} S=S^{[l]} \nabla^{l}$ is well known [20]. As observed in [19], offset invariance [18] is the right concept to characterize the existence of difference schemes in the nonlinear case.

Definition 6 [19] A binary subdivision operator $S$ is offset invariant (OSI) for $\Pi_{k}$ if for each $f \in l_{\infty}(\mathbb{Z})$ and any polynomial $P(x) \in \Pi_{m}, m \leq k$ there exists a polynomial, $Q$, of degree $<m$ such that

$$
S\left(f+\left.P\right|_{\mathbb{Z}}\right)=S f+\left.(P+Q)\right|_{2^{-1} \mathbb{Z}} .
$$

Schemes of the form (4) with $\delta=\nabla^{k}$ are offset invariant for $\Pi_{k-1}$. To check this, let $P(x) \in \Pi_{k-1}$. Since $\nabla^{k}\left(\left.P\right|_{\mathbb{Z}}\right)=0$, we have $\forall f \in l_{\infty}(\mathbb{Z})$

$$
\begin{aligned}
S_{\mathcal{N}}\left(f+\left.P\right|_{\mathbb{Z}}\right) & =S_{\mathcal{L}}\left(f+\left.P\right|_{\mathbb{Z}}\right)+\mathcal{F}\left(\nabla^{k} f\right)=S_{\mathcal{L}}(f)+\left.P\right|_{2^{-1} \mathbb{Z}}+\mathcal{F}\left(\nabla^{k} f\right) \\
& =S_{\mathcal{N}}(f)+\left.P\right|_{2^{-1} \mathbb{Z}} .
\end{aligned}
$$

It is proven in [19] that offset invariance for $\Pi_{k}$ guarantees the existence of the difference schemes $S^{[l]}$ for $l \leq k+1$. Thus the new families of nonlinear subdivision schemes are offset invariant for $\Pi_{1}$, which guarantees the existence of the first and second difference schemes. For the families in Eqs. 27-28, these schemes can be easily computed by elementary means. Introducing the restriction operator $(m<n)$

$$
\chi_{m, n}: l_{\infty}(\mathbb{Z}) \rightarrow \mathbb{R}^{n-m+1}, \quad \chi_{m, n}(f)=\left(f_{m}, f_{m+1}, \ldots, f_{n}\right),
$$

and the functions $L_{l, r}: \mathbb{R}^{4} \rightarrow \mathbb{R}$

$L_{3,1}(x)=-x_{1}+3 x_{2}, \quad L_{2,2}(x)=x_{2}+x_{3}, \quad L_{1,3}(x)=3 x_{3}-x_{4}, \quad x=\left(x_{1}, x_{2}, x_{3}, x_{4}\right) \in \mathbb{R}^{4}$,

so that

$$
\left(\mathcal{L}_{l, r} f\right)_{2 n+1}=-\frac{1}{16} L_{l, r} \circ \chi_{n-2, n+1} f, \quad(l, r)=(1,3),(3,1),(2,2),
$$


we can write $\forall f \in l_{\infty}(\mathbb{Z})$,

$$
\left\{\begin{array}{l}
\left(\mathrm{SWH}_{p, q} f\right)_{2 n}=f_{n} \\
\left(\mathrm{SWH}_{p, q} f\right)_{2 n+1}=\left(S_{1,1} f\right)_{2 n+1}+G_{p, q} \circ \chi_{n-2, n+1} \nabla^{2} f
\end{array}\right.
$$

with

$$
G_{p, q}(x)=-\frac{1}{16} \mathrm{~W}_{p, \frac{3}{8}, \frac{5}{8}}\left(\mathrm{H}_{q}\left(L_{3,1}(x), L_{1,3}(x)\right), L_{2,2}(x)\right) .
$$

From Eq. 29, it can be easily deduced that

$$
\left\{\begin{array}{l}
\left(\mathrm{SWH}_{p, q}^{[2]} w\right)_{2 n}=2 G_{p, q} \circ \chi_{n-2, n+1} w, \\
\left(\mathrm{SWH}_{p, q}^{[2]} w\right)_{2 n+1}=\frac{w_{n}}{2}+G_{p, q} \circ \chi_{n-2, n+1} w+G_{p, q} \circ \chi_{n-1, n+2} w .
\end{array}\right.
$$

We obtain a similar result for $\mathrm{SHW}_{q, p}$, substituting $G_{p, q}$ by $R_{q, p}$

$$
R_{q, p}(x)=-\frac{1}{16} \mathrm{H}_{q}\left(\mathrm{~W}_{p, \frac{3}{8}, \frac{5}{8}}\left(L_{3,1}(x), L_{2,2}(x)\right), \mathrm{W}_{p, \frac{3}{8}, \frac{5}{8}}\left(L_{1,3}(x), L_{2,2}(x)\right)\right) .
$$

We remark that the new schemes reproduce exactly $\Pi_{3}$, however they are only offset invariant for $\Pi_{1}$ and, hence, difference schemes $S^{[k]}$ do not exist for $k>2$.

\subsection{Convergence}

As observed in $[19,22]$, the existence of difference schemes may be very helpful in proving the convergence of a nonlinear scheme. Since $\delta=\nabla^{2}$,

$$
\delta S^{L}=\nabla^{2} S S^{L-1}=S^{[2]} \nabla^{2} S^{L-1}=\left(S^{[2]}\right)^{L} \nabla^{2},
$$

so that $\mathbf{C 2}$ in Theorem 1 is equivalent to the following condition

$$
\exists L>0,0<T<1: \quad\left\|\left(S^{[2]}\right)^{L}(f)\right\|_{\infty} \leq T\|f\|_{\infty} \quad \forall f \in l^{\infty}(\mathbb{Z}) .
$$

Theorem 7 The schemes $S W H_{p, q}$ and $S H W_{q, p}$ are uniformly convergent, for all $p, q \geq 1$.

Proof We shall check the conditions in Theorem 1. To check $\mathbf{C 1}$, we need to find a uniform bound for the non-linear functions $G_{p, q}$ and $R_{q, p}$ in Eqs. 30, Eq. 32. Using Eq. 24-(a) we get that $\forall x \in \mathbb{R}^{4}$

$$
\begin{aligned}
\left|G_{p, q}(x)\right| & \leq \frac{1}{16}\left|\frac{3}{8} \mathrm{H}_{q}\left(L_{3,1}(x), L_{1,3}(x)\right)+\frac{5}{8} L_{2,2}(x)\right| \leq \frac{11}{64}\|x\|_{\infty}, \\
\left|R_{q, p}(x)\right| & \leq \frac{1}{16}\left|\frac{1}{2}\left(\mathrm{~W}_{p, \frac{3}{8}, \frac{5}{8}}\left(L_{3,1}(x), L_{2,2}(x)\right)+\frac{1}{2} \mathrm{~W}_{p, \frac{3}{8}, \frac{5}{8}}\left(L_{1,3}(x), L_{2,2}(x)\right)\right)\right| \leq \frac{11}{64}\|x\|_{\infty} .
\end{aligned}
$$

To check C2 we consider its equivalent formulation (34). For even indexes,

$$
\left|\left(\mathrm{SWH}_{p, q}^{[2]} f\right)_{2 n}\right| \leq \frac{11}{32}\|f\|_{\infty}, \quad\left|\left(\mathrm{SHW}_{q, p}^{[2]} f\right)_{2 n}\right| \leq \frac{11}{32}\|f\|_{\infty},
$$


using the previously computed bounds. For odd components we get

$$
\max \left\{\left|\left(\operatorname{SWH}_{p, q}^{[2]} f\right)_{2 n+1}\right|,\left(\operatorname{SHW}_{q, p}^{[2]} f\right)_{2 n+1} \mid\right\} \leq \frac{1}{2}\|f\|_{\infty}+\frac{11}{64}\left(\|f\|_{\infty}+\|f\|_{\infty}\right) \leq \frac{27}{32}\|f\|_{\infty} .
$$

Hence,

$$
\max \left\{\left\|\mathrm{SWH}_{p, q}^{[2]}\right\|_{\infty},\left\|\mathrm{SHW}_{q, p}^{[2]}\right\|_{\infty}\right\} \leq \frac{27}{32}<1
$$

Remark We notice that the bound in Eq. 35 implies that for $S=\mathrm{SWH}_{p, q}, \mathrm{SHW}_{q, p}$ and $f \in l_{\infty}(\mathbb{Z})$ the limit function $S^{\infty} f$ is at least $C^{\beta-}$ with $\beta=$ $\min \left\{-\log _{2}\left(\frac{27}{32}\right), 1\right\} \simeq 0.2540$. This result appears to be suboptimal, since all numerical evidence suggests that $S^{\infty} f \in C^{1-}$.

\subsection{Order of accuracy}

The order of approximation of a subdivision scheme measures the approximation properties of the recursive process when applied to discrete data coming from smooth functions.

Definition 8 A convergent subdivision scheme $S$ has approximation order $r$ if

$$
\left\|S^{\infty} f^{0}-F\right\|_{\infty} \leq D h^{r}
$$

when $f_{i}^{0}=F(i h), i \in \mathbb{Z}$ for any $F(x)$ sufficiently smooth.

For a given subdivision scheme, the order of approximation after one iteration is usually much easier to obtain.

Definition 9 Let $S$ be a subdivision scheme that satisfies

$$
\max _{i}\left|f_{2 i+1}^{1}-F(i h+h / 2)\right| \leq C h^{r}, \quad C<\infty
$$

with $f^{1}=S f^{0}$ and $f_{i}^{0}=F(i h), i \in \mathbb{Z}$ for any $F(x)$ sufficiently smooth. Then $r$ is called the order of approximation after one iteration of $S$.

Obviously, the order of approximation after one iteration of interpolatory subdivision schemes based on Lagrange interpolation is at least as high as that of the interpolatory reconstruction used in its design. We notice that Eq. 10 implies that the order of approximation after one iteration of the Power $_{p}$ schemes is at least 4, when refining smooth convex functions and $p \geq 2$, since

$$
\left\|S_{\mathrm{H}_{p}} f-\left.F\right|_{2^{-1} h \mathbb{Z}}\right\|_{\infty} \leq\left\|S_{\mathrm{H}_{p}} f-S_{2,2} f\right\|_{\infty}+\left\|S_{2,2} f-\left.F\right|_{2^{-1} h \mathbb{Z}}\right\|_{\infty}=O\left(h^{4}\right) .
$$

For the schemes defined in this paper, we can also measure how close the new schemes are to the 6-point DD scheme for smooth convex functions. 
Remark 10 We know that if $f_{i}=F(i h)$ and $F(x)$ is smooth and convex, $\left(\nabla^{2} f\right)_{i}$ do not change sign. We can show, by straightforward Taylor expansions, that

$$
\left(\mathcal{L}_{l, r} \nabla^{2} f\right)_{i}=2 F^{\prime \prime}\left(x_{i}\right) h^{2}+F^{\prime \prime \prime}\left(x_{i}\right) h^{3}+O\left(h^{4}\right) \quad(l, r) \in\{(1,3),(3,1),(2,2)\}
$$

hence we also expect that, for smooth convex functions and $h$ small enough, $\left(\mathcal{L}_{l, r} \nabla^{2} f\right)_{i}$ will not change sign either.

Proposition 11 Let $F: \mathbb{R} \rightarrow \mathbb{R}$ a smooth function, and $f=\{F(i h)\}_{i \in \mathbb{Z} \text {. }}$ If $\left(\mathcal{L}_{l, r} \nabla^{2} f\right)_{n}$ have the same sign $\forall n \in \mathbb{Z},(l, r) \in\{(1,3),(3,1),(2,2)\}$, and $\left|F^{\prime \prime}(x)\right|>\rho>0, x \in \mathbb{R}$, then

$$
\left\|S_{3,3} f-S W H_{p, q} f\right\|_{\infty}=\mathcal{O}\left(h^{r}\right)=\left\|S_{3,3} f-S H W_{q, p} f\right\|_{\infty}, \quad r=\min \{2 p+2,3 q+2\} .
$$

Proof Notice that

$$
\left(S_{3,3} f\right)_{2 n+1}-\left(\mathrm{SWH}_{p, q} f\right)_{2 n+1}=-\frac{1}{16}\left(\operatorname{ave}_{\frac{3}{8}, \frac{5}{8}}\left(\operatorname{ave}_{\frac{1}{2}, \frac{1}{2}}(x, z), y\right)-\mathrm{W}_{p, \frac{3}{8}, \frac{5}{8}}\left(\mathrm{H}_{q}(x, z), y\right)\right)
$$

with

$$
x:=3 \nabla^{2} f_{n-1}-\nabla^{2} f_{n-2}, \quad y:=\nabla^{2} f_{n-1}+\nabla^{2} f_{n}, \quad z:=3 \nabla^{2} f_{n}-\nabla^{2} f_{n+1} .
$$

Since $F$ is a smooth function, the Taylor expansions in Eq. 39 show that $x, y, z$ are $O\left(h^{2}\right)$ and non-zero, provided that $h$ is sufficiently small. Since (24)-(b) ensures that $\mathrm{W}_{p, a, b}\left(O\left(h^{r}\right), O\left(h^{s}\right)\right)=O\left(h^{\min \{r, s\}}\right)$, we have that $\mathrm{H}_{q}(x, z)=\mathcal{O}\left(h^{2}\right)$, $\mathrm{W}_{p, a, b}(x, y)=\mathcal{O}\left(h^{2}\right)=\mathrm{W}_{p, a, b}(y, z)$. We write

$$
\operatorname{ave}_{\frac{3}{8}, \frac{5}{8}}\left(\operatorname{ave}_{\frac{1}{2}, \frac{1}{2}}(x, z), y\right)-\mathrm{W}_{p, \frac{3}{8}, \frac{5}{8}}\left(\mathrm{H}_{q}(x, z), y\right)=Z_{1}(x, y, z)+Z_{2}(x, y, z)
$$

with

$$
\begin{aligned}
& Z_{1}(x, y, z):=\operatorname{ave}_{\frac{3}{8}, \frac{5}{8}}\left(\operatorname{ave}_{\frac{1}{2}, \frac{1}{2}}(x, z), y\right)-\operatorname{ave}_{\frac{3}{8}, \frac{5}{8}}\left(\mathrm{H}_{q}(x, z), y\right)=\frac{3}{8}\left(\operatorname{ave}_{\frac{1}{2}, \frac{1}{2}}(x, z)-\mathrm{H}_{q}(x, z)\right) \\
& Z_{2}(x, y, z):=\operatorname{ave}_{\frac{3}{8}, \frac{5}{8}}\left(\mathrm{H}_{q}(x, z), y\right)-\mathrm{W}_{p, \frac{3}{8}, \frac{5}{8}}\left(\mathrm{H}_{q}(x, z), y\right) .
\end{aligned}
$$

Notice that (using Taylor expansions, when necessary)

$$
\begin{aligned}
& x-z=3 \nabla^{2} f_{n-1}-\nabla^{2} f_{n-2}-3 \nabla^{2} f_{n}+\nabla^{2} f_{n+1}=-\nabla^{4} f_{n-2}+\nabla^{4} f_{n-1}=\nabla^{5} f_{n-2}=O\left(h^{5}\right) \\
& x+z=3 \nabla^{2} f_{n-1}-\nabla^{2} f_{n-2}+3 \nabla^{2} f_{n}-\nabla^{2} f_{n+1}=O\left(h^{2}\right) .
\end{aligned}
$$

Hence, assuming without loss of generality that $x, z \geq 0$, Eq. 7 leads to

$$
Z_{1}(x, y, z)=\frac{3}{16} \frac{|x-z|^{q}}{|x+z|^{q-1}}=\mathcal{O}\left(h^{3 q+2}\right) .
$$

For $Z_{2}(x, y, z)$, denoting $s=H_{p}(x, z)$ and using Eq. 22, we get that $(s, y>0)$,

$$
Z_{2}(x, y, z)=\operatorname{ave}_{\frac{3}{8}, \frac{5}{8}}(s, y)-\mathrm{W}_{p, a, b}(s, y)=\left(\frac{3}{8} s+\frac{5}{8} y\right) \frac{|s-y|^{p}}{\left(M+\frac{3}{5} m\right)\left(M+\frac{5}{3} m\right)^{p-1}}
$$


with $M=\max \{s, y\}, m=\min \{s, y\}$. Notice that $s=O\left(h^{2}\right)$, hence

$$
\operatorname{ave}_{\frac{3}{8}, \frac{5}{8}}(s, y)=O\left(h^{2}\right), \quad M=\min \{s, y\}=O\left(h^{2}\right), \quad m=\max \{s, y\}=O\left(h^{2}\right) .
$$

\section{Moreover,}

$$
s-y=\mathrm{H}_{q}(x, z)-y=\frac{x-2 y+z}{2}-\frac{1}{2} \frac{|x-z|^{q}}{(x+z)^{q-1}}=\mathcal{O}\left(h^{4}\right)+\mathcal{O}\left(h^{3 q+2}\right),
$$

because

$$
x-2 y+z=-\nabla^{2} f_{n-2}+\nabla^{2} f_{n-1}+\nabla^{2} f_{n}-\nabla^{2} f_{n+1}=\nabla^{3} f_{n-2}-\nabla^{3} f_{n}=\nabla^{4} f_{n-2}=O\left(h^{4}\right) .
$$

Since $4<3 q+2$ for $q \geq 1$, we may conclude that

$$
Z_{2}(x, y, z)=O\left(h^{2}\right) O\left(h^{4 p-2 p}\right)=O\left(h^{2 p+2}\right),
$$

from which we deduce the desired result for the schemes $\mathrm{SWH}_{p, q}$.

For the $\mathrm{SHW}_{q, p}$ family, we proceed in a similar way. Assume $x, y, z>0$ and write

$$
\left(S_{3,3} f\right)_{2 n+1}-\left(\operatorname{SHW}_{q, p} f\right)_{2 n+1}=-\frac{1}{16}\left(Y_{1}(x, y, z)+Y_{2}(x, y, z)\right)
$$

with

$$
\begin{aligned}
Y_{1}(x, y, z) & =\operatorname{ave}_{\frac{1}{2}, \frac{1}{2}}\left(\operatorname{ave}_{\frac{3}{8}, \frac{5}{8}}(x, y), \operatorname{ave}_{\frac{3}{8}, \frac{5}{8}}(z, y)\right)-\operatorname{ave}_{\frac{1}{2}, \frac{1}{2}}\left(\mathrm{~W}_{p, \frac{3}{8}, \frac{5}{8}}(x, y), \mathrm{W}_{p, \frac{3}{8}, \frac{5}{8}}(z, y)\right), \\
& \left.=\frac{1}{2}\left(\operatorname{ave}_{\frac{3}{8}, \frac{5}{8}}(x, y)-\mathrm{W}_{p, \frac{3}{8}, \frac{5}{8}}(x, y)\right)+\frac{1}{2}\left(\operatorname{ave}_{\frac{3}{8}, \frac{5}{8}}(z, y)-\mathrm{W}_{p, \frac{3}{8}, \frac{5}{8}}(z, y)\right)\right) \\
Y_{2}(x, y, z) & \left.=\operatorname{ave}_{\frac{1}{2}, \frac{1}{2}}\left(\mathrm{~W}_{p, \frac{3}{8}, \frac{5}{8}}(x, y), \mathrm{W}_{p, \frac{3}{8}, \frac{5}{8}}(z, y)\right)-\mathrm{H}_{q}\left(\mathrm{~W}_{p, \frac{3}{8}, \frac{5}{8}}(x, y), \mathrm{W}_{p, \frac{3}{8}, \frac{5}{8}}(z, y)\right)\right) .
\end{aligned}
$$

As before, it is easy to deduce that

$$
\begin{aligned}
& x-y=-\nabla^{4} f_{n-2}=O\left(h^{4}\right), \quad x+y=O\left(h^{2}\right), \\
& z-y=-\nabla^{4} f_{n-1}=O\left(h^{4}\right), \quad z+y=O\left(h^{2}\right) .
\end{aligned}
$$

Hence, for $x, y, z \geq 0$, using Eq. 22 and proceeding as in Eq. 40 we get,

$$
\operatorname{ave}_{\frac{3}{8}, \frac{5}{8}}(x, y)-\mathrm{W}_{p, \frac{3}{8}, \frac{5}{8}}(x, y)=O\left(h^{2 p+2}\right)=\operatorname{ave}_{\frac{3}{8}, \frac{5}{8}}(z, y)-\mathrm{W}_{p, \frac{3}{8}, \frac{5}{8}}(z, y) .
$$

Thus $Y_{1}(x, y, z)=O\left(h^{2 p+2}\right)$ (notice that the two terms of the $\frac{1}{2}, \frac{1}{2}$ average in $Y_{1}$ have the same sign).

For $Y_{2}(x, y, z)$, we use that $\mathrm{W}_{p, a, b}: \mathbb{R}^{2} \rightarrow \mathbb{R}$ is a Lipschitz function (see next section), hence

$$
\left|\mathrm{W}_{p, \frac{3}{8}, \frac{5}{8}}(x, y)-\mathrm{W}_{p, \frac{3}{8}, \frac{5}{8}}(z, y)\right| \leq L|x-z|=O\left(h^{5}\right)
$$

being $L$ the Lipschitz constant of $\mathrm{W}_{p, \frac{3}{8}, \frac{5}{8}}$. Then, defining $u:=\mathrm{W}_{p, \frac{3}{8}, \frac{5}{8}}(x, y), v:=$ $\mathrm{W}_{p, \frac{3}{8}, \frac{5}{8}}(z, y)$ and noticing $u, v>0$ if $x, y, z>0$ and $u=O\left(h^{2}\right)=v$, we have, using Eqs. 7 and 41 ,

$Y_{2}(x, y, z)=\operatorname{ave}_{\frac{1}{2}, \frac{1}{2}}(u, v)-\mathrm{H}_{q}(u, v)=\frac{1}{2} \frac{|u-v|^{q}}{|u+v|^{q-1}}=\frac{O\left(h^{5 q}\right)}{O\left(h^{2 q-2}\right)}=O\left(h^{3 q+2}\right)$,

from which we deduce the desired result also for the schemes $\mathrm{SHW}_{q, p}$. 
Corollary 12 Under the same conditions as in the previous proposition

$$
\left\|S W H_{p, q} f-\left.F\right|_{2^{-1} h \mathbb{Z}}\right\|_{\infty}=O\left(h^{r}\right)=\left\|S H W_{q, p} f-\left.F\right|_{2^{-1} h \mathbb{Z}}\right\|_{\infty}, \quad r=\min \{2 p+2,3 q+2,6\} .
$$

As shown in [5], the order of approximation of a stable subdivision scheme can be deduced from its order of approximation after one iteration. The following result from [5] holds for linear as well as nonlinear subdivision schemes and it serves also as a motivation to study the stability of the nonlinear schemes under consideration.

Theorem 13 Let $S$ be a convergent subdivision scheme whose approximation order after one iteration is $r \geq 1$. Then if $S$ is stable, it has approximation order $r$.

In [19] it is shown that the Power $_{p}$ schemes are stable subdivision schemes for $p<3$ and unstable for $p \geq 4$, hence (38) and Theorem 13 ensure that the order of approximation of the Power $_{p}$ schemes is 4, when refining smooth convex functions, for $p<3$.

In the following section we examine the question of stability for the families of schemes (27) and Eq. 28, in order to check whether or not similar conclusions can be extracted for the new families of schemes presented in this paper.

\section{Stability of the 6-point nonlinear schemes}

Theorem 1 establishes that stability follows from two facts: Lipschitz-continuity of the operator $\mathcal{F}$ and contractivity of $\delta S_{\mathcal{N}}^{L}$, for some $L>0$. When $\delta=\nabla^{2}$ and $S_{\mathcal{N}}$ is offset invariant for $\Pi_{1}$ (the case of the new families of 6-point schemes), condition S2 can be equivalently expressed as follows

$$
\exists L>0,0<\mu<1:\left\|\left(S_{\mathcal{N}}^{[2]}\right)^{L} f-\left(S_{\mathcal{N}}^{[2]}\right)^{L} g\right\|_{\infty}=\mu\|f-g\|_{\infty}, \forall f, g \in l_{\infty}(\mathbb{Z}) .
$$

We shall see next that the second difference schemes in Eq. 31 are defined by nonlinear functions that admit uniformly bounded Generalized Gradients. In [22], this fact was used to show the stability of a nonlinear, monotonicity preserving, scheme by expressing the contractivity condition (42) in terms of the Generalized Jacobian of the scheme and using Corollary 24 in the Appendix (or see [19]). However, we shall see that this technique does not seem to be as useful for the schemes considered in this paper.

The first step is to show that the Weighted-Power $p$ mean (22) belongs to a special class of continuous, piecewise smooth functions: the class of $C_{p w}^{1}\left(\mathbb{R}^{2}\right)$ functions. Functions in this class are continuous, piecewise smooth and have uniformly bounded directional derivatives except (maybe) at $0 \in \mathbb{R}^{m}$ and across certain hyperplanes separating regions of $C^{1}$ smoothness. Directional derivatives along the separating hyperplanes do, also, exist. For this class of functions it is possible to define a Generalized Gradient using only the gradients on smooth regions. As the classical gradient 
for smooth functions, the linear map associated to any Generalized Gradient recovers all directional derivatives that 'make sense', and satisfies a chain rule property for the composition with Lipschitz curves. We refer the reader to [19] or the Appendix to this paper for the definition, and the main properties, of this class of functions.

\subsection{The Weighted-Harmonic mean: Generalized Gradients}

Property (24)-(a) implies that $\mathrm{W}_{p, a, b}(x, y)$ is a continuous function in $\mathbb{R}^{2}$. It is obviously differentiable in the interior of the sectors in $\mathbb{R}^{2}$ separated by the three hyperplanes $\mathcal{H}_{1}=\{x=0\}, \mathcal{H}_{2}=\{y=0\}, \mathcal{H}_{3}=\{x=y\}$. As observed in [19] (see also the Appendix) a Generalized Gradient for $\mathrm{W}_{p, a, b}(x, y)$ can be defined using only the gradients in smoothness regions (see Eq. A.3), provided that certain compatibility conditions are satisfied over the separating hyperplanes. Since $\mathrm{W}_{p, a, b}(x, y)=-\mathrm{W}_{p, a, b}(-x,-y)$, it is enough to consider the half plane $y \geq 0$. Then, the compatibility conditions (A.2) amount to showing that

$$
\begin{aligned}
\lim _{0 \neq x>y \rightarrow 0} \nabla \mathrm{W}_{p, a, b}(x, y) \cdot(1,0) & =0, \quad \lim _{0 \neq y>x \rightarrow 0} \nabla \mathrm{W}_{p, a, b}(x, y) \cdot(0,1)=0, \\
\lim _{y>x \neq 0,(x, y) \rightarrow(d, d)} \nabla \mathrm{W}_{p, a, b}(x, y) \cdot(1,1) & =\left(\mathrm{W}_{p, a, b}(x, x)\right)^{\prime}=1, \quad \forall d>0, \\
\lim _{0 \neq y<x,(x, y) \rightarrow(d, d)} \nabla \mathrm{W}_{p, a, b}(x, y) \cdot(1,1) & =\left(\mathrm{W}_{p, a, b}(x, x)\right)^{\prime}=1, \quad \forall d>0 .
\end{aligned}
$$

To check these conditions, we first notice that $\mathrm{W}_{p, b, a}(y, x)=\mathrm{W}_{p, a, b}(x, y)$, hence it is enough to consider the gradients of the 1-homogeneous ${ }^{2}$ function (see Fig. 7)

$$
\phi_{p, a, b}(x, y)=(a x+b y)\left(1-\frac{(y-x)^{p}}{\left(y+\frac{1}{\alpha} x\right)(y+\alpha x)^{p-1}}\right), \quad \alpha=\max (a, b) / \min (a, b) .
$$

A straightforward computation leads to

$$
\begin{aligned}
\nabla \phi_{p, a, b}(x, y) & =(y,-x) \rho_{p, a, b}(x, y)+(a, b) \sigma_{p, \alpha}(x, y), \\
\rho_{p, a, b}(x, y) & =\frac{\alpha(\alpha+1)(y-x)^{p-1}(a x+b y)(x(\alpha+p-1)+y(\alpha(p-1)+1))}{(x+\alpha y)^{2}(\alpha x+y)^{p}}, \\
\sigma_{p, \alpha}(x, y) & =1-\frac{\alpha(y-x)^{p}}{(x+\alpha y)(\alpha x+y)^{p-1}} .
\end{aligned}
$$

Since $\nabla \phi_{a, b}$ is 0-homogeneous, for $y>0, \nabla \phi_{a, b}(x, y)=\nabla \phi_{a, b}(x / y, y / y)=$ $\nabla \phi_{a, b}(t, 1), t=x / y$, hence

$$
\nabla \phi_{a, b}(t, 1)=\mu_{p, a, b}(t)(1,-t)+\eta_{p, \alpha}(t)(a, b) .
$$

with

\footnotetext{
${ }^{2}$ A function $F$ is $n$-homogeneous if $F(\lambda x)=\lambda^{n} F(x)$, being $x$ a vector and $\lambda$ a scalar.
} 


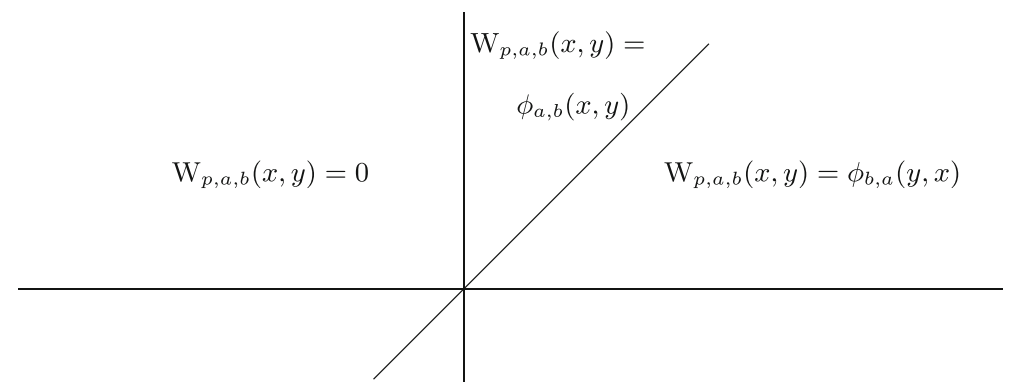

Fig. $7 \mathrm{~W}_{p, a, b}(x, y)$ in its smoothness sectors

$$
\begin{gathered}
\rho_{p, a, b}(x, y)=\mu_{p, a, b}(x / y), \quad \mu_{p, a, b}(t):=\frac{c_{1}(1-t)^{p-1}(a t+b)\left(c_{2} t+c_{3}\right)}{(t+\alpha)^{2}(\alpha t+1)^{p}}\left\{\begin{array}{l}
c_{1}=\alpha(\alpha+1) \\
c_{2}=\alpha+p-1 \\
c_{3}=\alpha(p-1)+1
\end{array},\right. \\
\sigma_{p, \alpha}(x, y)=\eta_{p, \alpha}(x / y), \quad \eta_{p, \alpha}(t):=1-\frac{\alpha(1-t)^{p}}{(t+\alpha)(\alpha t+1)^{p-1}} .
\end{gathered}
$$

Clearly, the functions $\mu_{p, a, b}(t), t \mu_{p, a, b}(t)$, are continuous in $[0,1]$. Moreover,

$$
\begin{aligned}
& \lim _{t \rightarrow 0} t \mu_{p, a, b}(t)=0, \quad \lim _{t \rightarrow 0} \mu_{p, a, b}(t)=\frac{c_{1} b c_{3}}{\alpha^{2}}, \quad \lim _{t \rightarrow 0} \eta_{p, \alpha}(t)=0, \\
& \lim _{t \rightarrow 1} \mu_{p, a, b}(t)=\lim _{t \rightarrow 1} t \mu_{p, a, b}(t)=\left\{\begin{array}{ll}
c_{1}\left(c_{2}+c_{3}\right) / \alpha^{2} & p=1 \\
0 & p>1
\end{array}, \quad \lim _{t \rightarrow 1} \eta_{p, \alpha}(t)=1 .\right.
\end{aligned}
$$

Taking into account Fig. 7, we observe that the compatibility conditions (43), Eq. 44, Eq. 45 follow from the fact that $\forall p \geq 1, \forall a, b \geq 0, a+b=1$,

$$
\begin{aligned}
\lim _{0 \neq y>x \rightarrow 0} \nabla \phi_{p, a, b}(x, y) \cdot(0,1) & =\lim _{t \rightarrow 0}-t \mu_{p, a, b}(t)+b \eta_{p, \alpha}(t)=0, \\
\lim _{y>x, x, y \rightarrow d \neq 0} \nabla \phi_{p, a, b}(x, y) \cdot(1,1) & =\lim _{t \rightarrow 1}(1-t) \mu_{p, a, b}(t)+(a+b) \eta_{p, \alpha}(t)=1 .
\end{aligned}
$$

In addition, $\nabla \phi_{a, b}(x, y)$ is uniformly bounded in $\{(x, y): y>x>0\}$ because $\nabla \phi_{a, b}(t, 1)$ is bounded for $0 \leq t \leq 1$. Hence, $\nabla \mathrm{W}_{p, a, b}(x, y)$ is also uniformly bounded for $(x, y)$ in any region of smoothness and, thus, $\mathrm{W}_{p, a, b}(x, y)$ in Eq. 22 belongs to the space $C_{p w}^{1}\left(\mathbb{R}^{2}\right)$. From Corollary $19, \mathrm{~W}_{p, a, b}(x, y)$ is a Lipschitz function. However, uniform bounds for $\left\|D \mathrm{~W}_{p, a, b}(x, y)\right\|$ depend on the parameters $p, a, b$ in a more involved way than for the Power $_{p}$ averages. We illustrate the required computations by examining the cases $p=1,2$.

Proposition $14 W_{1, a, b}(x, y)$ admits a generalized gradient, which satisfies $\forall(x, y) \in \mathbb{R}^{2}$

$$
\left\|D W_{1, a, b}(x, y)\right\|_{1} \leq 1+2(c-d), \quad c=\max \{a, b\}, d=\min \{c, d\}
$$


Proof For $p=1$,

$$
\mu_{1, a, b}(t)=\frac{\alpha(\alpha+1)(a t+b)}{(t+\alpha)^{2}}, \quad \eta_{1, \alpha}(t)=1-\frac{\alpha(1-t)}{t+\alpha}=(1+\alpha) \frac{t}{t+\alpha} .
$$

Since $\alpha=c / d$, and $a+b=c+d=1$, Eq. 46 becomes

$$
\nabla \phi_{1, a, b}(t, 1)=c \frac{a t+b}{(d t+c)^{2}}(1,-t)+\frac{t}{d t+c}(a, b) .
$$

If $d=a \leq b=c$, we get

$$
\nabla \phi_{1, a, b}(t, 1)=\frac{c}{(d t+c)}(1,-t)+\frac{t}{d t+c}(d, c)=(1,0) \quad \Rightarrow \quad\left\|\nabla \phi_{1, a, b}(t, 1)\right\|_{1}=1 .
$$

If $d=b \leq a=c$, after some algebraic manipulations (notice that $c^{2}-d^{2}=c-d$ ) we get

$$
\nabla \phi_{1, a, b}(x, y)=(1,0)+\frac{c-d}{(d t+c)^{2}}\left(d t^{2}+2 c t-c,-t^{2}\right)
$$

hence $\left\|\nabla \phi_{1, a, b}(t, 1)\right\|_{1}=1+\frac{c-d}{(d t+c)^{2}}\left(d t^{2}+2 c t-c+1\right)$. This is an increasing function in $[0,1]$, hence

$$
1 \leq\left\|\nabla \phi_{1, a, b}(0,1)\right\|_{1} \leq\left\|\nabla \phi_{1, a, b}(t, 1)\right\|_{1} \leq\left\|\nabla \phi_{1, a, b}(1,1)\right\|_{1}=1+2(c-d)
$$

which proves the result.

Proposition $15 W_{2, a, b}$ admits a generalized gradient that satisfies $\forall(x, y) \in \mathbb{R}^{2}$

$$
0 \leq D_{1} W_{2, a, b}(x, y) \leq \frac{1}{a}, \quad 0 \leq D_{2} W_{2, a, b}(x, y) \leq \frac{1}{b}, \quad\left\|D W_{2, a, b}(x, y)\right\|_{1} \leq \frac{1}{d}
$$

Proof We proceed as in Proposition 14. After straightforward manipulations we get

$$
\nabla \phi_{2, a, b}(t, 1)=\frac{1}{(b t+a)^{2}}\left(a, b t^{2}\right) .
$$

Notice that $D_{1} \phi_{2, a, b}(t, 1)=a /(b t+a)^{2}$ is a decreasing function in [0,1] while $D_{2} \phi_{2, a, b}(t)=b t^{2} /(b t+a)^{2}$ is increasing. Moreover, both components are positive, hence $\left\|\nabla \phi_{2, a, b}(t)\right\|_{1}=D_{1} \phi_{2, a, b}(t)+D_{2} \phi_{2, a, b}(t)=\left(a+b t^{2}\right)(b t+a)^{2}$, which is a decreasing function. Hence we readily conclude

$$
a \leq D_{1} \phi_{2, a, b}(t, 1) \leq \frac{1}{a}, \quad 0 \leq D_{2} \phi_{2, a, b}(t, 1) \leq b, \quad 1 \leq\left\|\nabla \phi_{2, a, b}(t, 1)\right\|_{1} \leq \frac{1}{a},
$$

and we deduce (49) from the relations above and Fig. 7.

Remark 16 We may also obtain specific bounds for the components of $D \mathrm{~W}_{1, a, b}(x, y)$, although the bounds are not as simple as for $p=2$. It is easy to 
see that the function $1+\frac{c-d}{(d t+c)^{2}}\left(d t^{2}+2 c t-c\right)$ is increasing and $\frac{c-d}{(d t+c)^{2}}\left(-t^{2}\right)$ is decreasing in $[0,1]$. Therefore $\forall t \in(0,1)$

$$
\begin{gathered}
D_{1} \phi_{1, a, b}(0,1) \leq D_{1} \phi_{1, a, b}(t, 1) \leq D_{1} \phi_{1, a, b}(1,1), \quad D_{2} \phi_{1, a, b}(1,1) \leq D_{2} \phi_{1, a, b}(t, 1) \leq D_{2} \phi_{1, a, b}(0,1) \\
\frac{d}{c} \leq D_{1} \phi_{1, a, b}(x, y) \leq 1+(c-d), \quad-(c-d) \leq D_{2} \phi_{1, a, b}(x, y) \leq 0, \quad \text { if } a>b . \quad
\end{gathered}
$$

From these bounds and Fig. 7, we may deduce general, uniform, bounds for the components of $D \mathrm{~W}_{1, a, b}(x, y)$.

\subsection{The Generalized Jacobian of the second difference schemes}

Let us consider the family of schemes $\mathrm{SWH}_{p, q}$ in Eq. 31. As specified in the Appendix, in order to define a Generalized Jacobian of $\mathrm{SWH}_{p, q}^{[2]}$ we need to justify the existence of uniformly bounded Generalized Gradients for the function $G_{p, q}$ in Eq. 30, which satisfy the chain rule (A.10).

Proposition 17 Let $\gamma:[a, b] \rightarrow \mathbb{R}^{m}$ be a Lipschitz curve. Then $\tilde{\gamma}=G_{p, q} \circ \gamma$ : $[a, b] \rightarrow \mathbb{R}$ is also Lipschitz and

$$
\tilde{\gamma}^{\prime}(t)=D G_{p, q}(\gamma(t)) \gamma^{\prime}(t) \quad \text { a.e. in }[a, b]
$$

where, $\forall x \in \mathbb{R}^{4}$,

$$
D G_{p, q}(x):=-\frac{1}{16} D W_{p, \frac{3}{8}, \frac{5}{8}}(\xi)\left(\begin{array}{cc}
D H_{q}(\rho) & 0 \\
(0,0) & 1
\end{array}\right)\left(\begin{array}{cccc}
-1 & 3 & 0 & 0 \\
0 & 0 & 3 & -1 \\
0 & 1 & 1 & 0
\end{array}\right),
$$

with $\rho=\left(L_{3,1}(x), L_{1,3}(x)\right), \quad \xi=\left(H\left(L_{3,1}(x), L_{1,3}(x)\right), L_{2,2}(x)\right)$.

Proof Notice that $G_{p, q}=-\frac{1}{16} \mathrm{~W}_{p, \frac{3}{8}, \frac{5}{8}} \circ \psi \circ M$ where $\psi: \mathbb{R}^{3} \rightarrow \mathbb{R}^{2}$ and $M: \mathbb{R}^{4} \rightarrow$ $\mathbb{R}^{3}$ are as follows

$$
\psi(x)=\left(\mathrm{H}_{q}\left(x_{1}, x_{2}\right), x_{3}\right), \quad M=\left(\begin{array}{cccc}
-1 & 3 & 0 & 0 \\
0 & 0 & 3 & -1 \\
0 & 1 & 1 & 0
\end{array}\right) .
$$

Thus,

$$
\tilde{\gamma}=G_{p, q} \circ \gamma=\mathrm{W}_{p, \frac{3}{8}, \frac{5}{8}} \circ \gamma_{2}, \quad \gamma_{2}:=\psi \circ \gamma_{1}, \quad \gamma_{1}:=M \circ \gamma
$$

Since $\gamma:[a, b] \rightarrow \mathbb{R}^{4}$ is Lipschitz, we have that a.e. in $(a, b)$

1. $\gamma_{1}:[a, b] \rightarrow \mathbb{R}^{3}$ is Lipschitz and $\gamma_{1}^{\prime}(t)=M \gamma^{\prime}(t)$,

2. $\gamma_{2}:[a, b] \rightarrow \mathbb{R}^{2}$ is Lipschitz and $\gamma_{2}^{\prime}(t)=D \psi\left(\gamma_{1}(t)\right) \gamma_{1}^{\prime}(t)$, with (see Theorem 22 in the Appendix)

$$
D \psi(x)=\left(\begin{array}{cc}
D \mathrm{H}_{q}\left(x_{1}, x_{2}\right) & 0 \\
(0,0) & 1
\end{array}\right), \quad \forall x=\left(x_{1}, x_{2}, x_{3}\right) \in \mathbb{R}^{3}
$$


3. $\tilde{\gamma}=\mathrm{W}_{p, \frac{3}{8}, \frac{5}{8}} \circ \gamma_{2}$ is Lipschitz (see Theorem 20 in the Appendix) $\tilde{\gamma}^{\prime}(t)=$ $D \mathrm{~W}_{p, \frac{3}{8}, \frac{5}{8}}\left(\gamma_{2}(t)\right) \gamma_{2}^{\prime}(t)$.

Collecting all of the above we have

$$
\tilde{\gamma}^{\prime}(t)=D \mathrm{~W}_{p, \frac{3}{8}, \frac{5}{8}}(\psi \circ M \circ \gamma(t)) D \psi(M \circ \gamma(t)) M \gamma^{\prime}(t), \quad \text { a.e. }(0,1)
$$

so that Eq. 52 provides an adequate definition of a Generalized Jacobian of $G_{p, q}$.

Uniform bounds for the Generalized Jacobian $D G_{p, q}$ defined in Eq. 52 can be readily computed. Since

$$
D G_{p, q}(x)=-\frac{1}{16} D \mathrm{~W}_{p, \frac{3}{8}, \frac{5}{8}}(\xi)\left(\begin{array}{c|c|c}
-D_{1} \mathrm{H}_{q}(\rho) & 3 D \mathrm{H}_{q}(\rho) & -D_{2} \mathrm{H}_{q}(\rho) \\
\hline 0 & (1,1) & 0
\end{array}\right),
$$

$\xi=\left(H\left(L_{3,1}(x), L_{1,3}(x)\right), L_{2,2}(x)\right), \rho=\left(L_{3,1}(x), L_{1,3}(x)\right)$, we can write

$$
\begin{gathered}
16 D_{1} G_{p, q}(x)=D_{1} \mathrm{~W}_{p, \frac{3}{8}, \frac{5}{8}}(\xi) D_{1} \mathrm{H}_{q}(\rho), \quad 16 D_{4} G_{p, q}(x)=D_{1} \mathrm{~W}_{p, \frac{3}{8}, \frac{5}{8}}(\xi) D_{2} \mathrm{H}_{q}(\rho), \\
16\left(D_{2} G_{p, q}(x), D_{3} G_{p, q}(x)\right)=-3 D_{1} \mathrm{~W}_{p, \frac{3}{8}, \frac{5}{8}}(\xi) D \mathrm{H}_{q}(\rho)-D_{2} \mathrm{~W}_{p, \frac{3}{8}, \frac{5}{8}}(\xi)(1,1)
\end{gathered}
$$

Taking into account that (see [19])

$$
0 \leq D_{1} \mathrm{H}_{q}(x) \leq q, \quad 0 \leq D_{2} \mathrm{H}_{q}(x) \leq q, \quad\left\|D \mathrm{H}_{q}(x)\right\|_{1} \leq q, \quad \forall x \in \mathbb{R}^{2},
$$

we have

$\left\|D G_{p, q}(x)\right\|_{1} \leq \frac{1}{16}\left(4\left|D_{1} \mathrm{~W}_{p, \frac{3}{8}, \frac{5}{8}}(\xi)\right| \cdot\left\|D \mathrm{H}_{q}(\rho)\right\|_{1}+2\left|D_{2} \mathrm{~W}_{p, \frac{3}{8}, \frac{5}{8}}(\xi)\right|\right) \leq \frac{q}{4}\left|D_{1} \mathrm{~W}_{p, \frac{3}{8}, \frac{5}{8}}(\xi)\right|+\frac{1}{8}\left|D_{2} \mathrm{~W}_{p, \frac{3}{8}, \frac{5}{8}}(\xi)\right|$.

As observed in the Appendix, the bi-infinite matrix with the following non-zero entries $\forall n \in \mathbb{Z}$

$$
\begin{aligned}
\left(D \mathrm{SWH}_{p, q}^{[2]}(w)\right)_{[2 n, n-2: n+1]} & =2 D G_{p, q}\left(\chi_{n-2, n+1} w\right), \\
\left(D \mathrm{SWH}_{p, q}^{[2]}(w)\right)_{[2 n+1, n-2: n+2]} & =\frac{1}{2}(0,0,1,0,0)-\left(D G_{p, q}\left(\chi_{n-2, n+1} w\right)+D G_{p, q}\left(\chi_{n-1, n+2} w\right)\right)
\end{aligned}
$$

defines a Generalized Jacobian of the second difference scheme. Then,

$$
\begin{aligned}
\left\|D\left(\mathrm{SWH}_{p, q}^{[2]}\right)_{[2 n,:]}(w)\right\|_{1} & \leq \frac{q}{2}\left|D_{1} \mathrm{~W}_{p, \frac{3}{8}, \frac{5}{8}}(\xi)\right|+\frac{1}{4}\left|D_{2} \mathrm{~W}_{p, \frac{3}{8}, \frac{5}{8}}(\xi)\right|, \\
\left\|D\left(\mathrm{SWH}_{p, q}^{[2]}\right)_{[2 n+1,:]}(w)\right\|_{1} & \leq \frac{1}{2}+\left\|D\left(\mathrm{SWH}_{p, q}^{[2]}\right)_{[2 n,:]}(w)\right\|_{1} .
\end{aligned}
$$

The strategy advocated in [22] for establishing stability relies on the relations (A.19), Eq. A.20 and Corollary 24, in the Appendix. The desired contractivity property (42) follows from the ability to find a uniform bound for products of Generalized Jacobians of the second difference scheme.

Taking into account the bounds obtained previously for the generalized gradient of the function $\mathrm{W}_{p, a, b}(x, y)$ for $p=1,2$ (these bounds are optimal for $p=2$ ) and the 
known (optimal) bounds for $\mathrm{H}_{q}(x, y)$, we display in Table 1 the values of the bound in Eq. 54 for $1 \leq p, q \leq 2$.

Since ( $S$ represents any scheme in the family)

$$
\left\|D S^{[2]}\right\|_{\infty}=\max _{n}\left\{\left\|\left(D S^{[2]}\right)_{[2 n,:]}\right\|_{1},\left\|\left(D S^{[2]}\right)_{[2 n+1,:]}\right\|_{1}\right\},
$$

from Eq. 54, Eq. 55 and the results in Table 1, we cannot ensure $\left\|D S^{[2]}\right\|_{\infty}<1$ for any member of our family of schemes, or, equivalently, we cannot ensure the contractivity of the second difference scheme.

As observed in [22], the technique described in the Appendix will be successful when the 1-norm of some row of the Generalized Jacobian is strictly uniformly bounded by 1 . In this case, and by carefully considering the form of the matrix products, it may be possible to arrive at products of Generalized Jacobians whose norms are strictly bounded by 1 . Taking into account the bounds in Table 1 , it seems that this strategy might only be feasible for $p=q=1$, because such case is the only one where Eq. 54 is strictly bounded by 1 . Since the task of obtaining theoretically bounds for products of Generalized Jacobian is very involved, we examine the issue numerically in the following section.

\section{Numerical experiments}

In this section we carry out a series of numerical experiments that illustrate the theoretical developments of the previous sections. We consider first the issue of the stability of the new schemes, from a numerical perspective. In addition, we also consider the smoothness of limit functions, as well as the approximation order of the non-oscillatory 6-point schemes, comparing the numerical results with those obtained for the Power $p$ schemes.

\subsection{Stability}

To examine the question of stability for each chosen subdivision scheme, $S$, we compute the quantities $C_{S}^{j}(h)$ for each $j \geq 1,0<h<1$,

$$
C_{S}^{j}(h) \approx \sup _{f^{0}} \sup _{\|\theta\|_{\infty}=1} \frac{1}{h}\left\|S^{j}\left(f^{0}+h \theta\right)-S^{j}\left(f^{0}\right)\right\|, \quad h>0 .
$$

For the computation we consider a sufficiently large set of sequences $f^{0}=\left\{f_{i}^{0}\right\}$ and perturbation sequences $\theta=\left\{\theta_{i}\right\}$, with components randomly chosen from the set $\{-1,0,1\}$ (hence $\|\theta\|_{\infty}=1$ ). We notice that if $S$ is Lipschitz stable, $C_{S}^{j}(h) \leq C$,

Table 1 Bounds of $\left\|D\left(\mathrm{SWH}_{p, q}^{[2]}\right)_{[2 n,:]}\right\|_{1}$

\begin{tabular}{lll}
\hline$p \backslash q$ & 1 & 2 \\
\hline 1 & $13 / 16$ & $21 / 16$ \\
2 & $52 / 30$ & $92 / 30$ \\
\hline
\end{tabular}



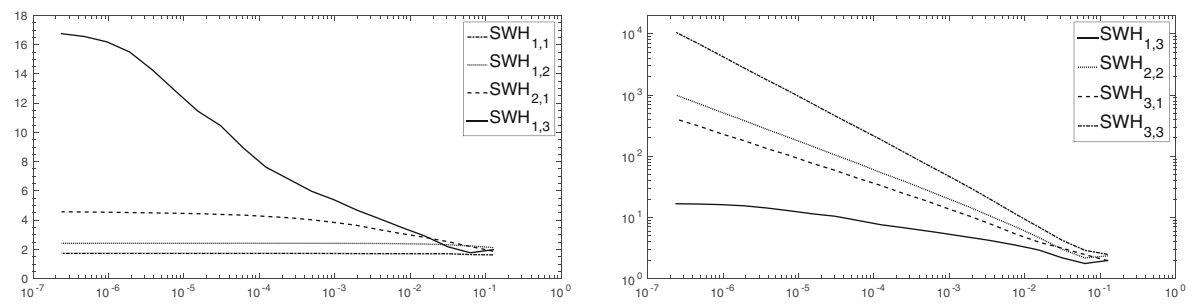

Fig. 8 Plot of $C_{S}^{L}(h)$, with respect to $h$ for $L=10, S=\mathrm{SWH}_{p, q}$ for several values of $p, q$

$\forall j \geq 1, \forall h>0$, so that any deviation with respect to this behavior may be considered a sign of the instability of the scheme.

Figure 8 displays $C_{S}^{10}(h)$ as a function of $h$, for $S=\mathrm{SWH}_{p, q}$ and various values of $p, q$. The plots in Fig. 8 seem to indicate that the scheme $\mathrm{SWH}_{p, q}$ is not stable for $p+q>3$.

As observed in Theorem 1, Lipschitz stability follows from the contractivity of an appropriate power of the second difference scheme, which is a condition that can also be examined numerically. For $h=10^{-7}$, the smallest value of $h$ considered in Fig. 8, we compute

$$
T_{S}^{j}(h) \approx \sup _{f^{0}} \sup _{\|\theta\|_{\infty}=1} \frac{1}{h}\left\|\left(S^{[2]}\right)^{j}\left(f^{0}+h \theta\right)-\left(S^{[2]}\right)^{j}\left(f^{0}\right)\right\|, \quad h>0,
$$

in order to check if the hypothesis $\mathbf{S 2}$, in its equivalent formulation (42), is fulfilled.

In Fig. 9 we display the values of $T_{S}^{j}(h)$ for $1 \leq j \leq 6$. We clearly notice that $\exists L \geq 1$ such that $T_{S}^{L}(h)<1$ for $S=\operatorname{SWH}_{p, q},(p, q)=(1,1),(1,2)$, a behavior that would be obtained if these schemes were stable. On the other hand, $T_{S}^{j}(h)$ appears to grow with $j$ for $p+q \geq 4$ indicating that condition (42) is not fulfilled.

We also observe that the $T_{S}^{j}$ does not grow for $S=\mathrm{SWH}_{2,1}$, but it does not appear to become smaller than one, an indication that condition (42) is only a sufficient condition for stability.

Table 2 summarizes the observations that might be deduced from our testing process. The same experiments were performed for the $\mathrm{SHW}_{q, p}$ family, with similar results.
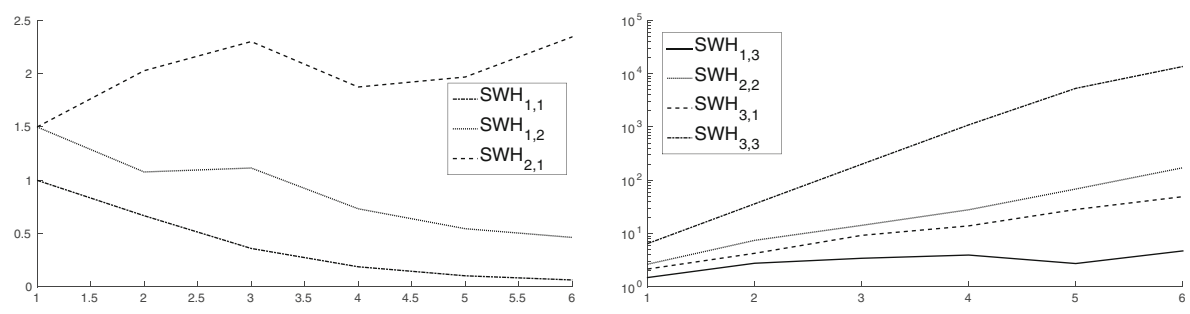

Fig. 9 Plot of $\left(T_{S}^{[2]}(h)\right)^{j}$ with respect to $j$ for $S=\mathrm{SWH}_{p, q}, h=10^{-7}$ and several values of $p, q$ 
Table 2 Stability perspective on $\mathrm{SWH}_{p, q}$ and $\mathrm{SHW}_{q, p} \cdot \checkmark$ suspected stability. $\times$ unstability

\begin{tabular}{llll}
\hline$q \backslash p$ & 1 & 2 & 3 \\
\hline 1 & $\checkmark$ & $\checkmark$ & $\times$ \\
2 & $\checkmark$ & $\times$ & $\times$ \\
3 & $\times$ & $\times$ & $\times$ \\
\hline
\end{tabular}

\subsection{Smoothness of the limit function}

In Section 3.2 we have shown that the schemes proposed in this paper are convergent, i.e. $S^{\infty} f^{0}$ is a continuous function $\forall f^{0} \in l_{\infty}(\mathbb{Z})$ for $S=\mathrm{SWH}_{p, q}, \mathrm{SHW}_{q, p}$. The regularity of the limit function obtained from Theorem 1 (see Remark 10) seems to be much smaller than what is observed in practice. In this section we perform a numerical study of the regularity of the proposed schemes based on the following observations (see [5]): Let us assume that $f(x)=S^{\infty} f^{0} \in C^{r-}, S$ an interpolatory subdivision scheme, and $r=l+\beta, l \in \mathbb{N}, 0 \leq \beta<1$. Then,

$$
f^{(l)}\left(x_{i+1}^{k}\right)-f^{(l)}\left(x_{i}^{k}\right) \approx C\left(x_{i+1}^{k}-x_{i}^{k}\right)^{\beta}=C h_{k}^{\beta} .
$$

Since $f_{i}^{k}=f\left(x_{i}^{k}\right)$, and $f^{(l)}\left(x_{i}^{k}\right) \approx \nabla^{l} f_{i}^{k} /\left(h_{k}\right)^{l}=2^{l k} \nabla^{l} f_{i}^{k} / h_{0}$, we have

$$
f^{(l)}\left(x_{i+1}^{k}\right)-f^{(l)}\left(x_{i}^{k}\right) \approx 2^{l k}\left(\nabla^{l} f_{i+1}^{k}-\nabla^{l} f_{i}^{k}\right) / h_{0}=2^{l k} \nabla^{l+1} f_{i}^{k} / h_{0} .
$$

Hence, we expect that

$$
\frac{2^{l k} \nabla^{l+1} f_{i}^{k}}{2^{l(k+1)} \nabla^{l+1} f_{i}^{k+1}} \approx \frac{h_{k}^{\beta}}{h_{k+1}^{\beta}}=2^{\beta} \quad \leftrightarrow \quad \frac{\nabla^{l+1} f_{i}^{k}}{\nabla^{l+1} f_{i}^{k+1}} \approx 2^{l+\beta} \leftrightarrow r=l+\beta \approx \log _{2}\left(\frac{\left\|\nabla^{l+1} f^{k}\right\|_{\infty}}{\left\|\nabla^{l+1} f^{k+1}\right\|_{\infty}}\right) .
$$

Therefore, in order to estimate the numerically regularity of a subdivision scheme, $S$, in a given region, $[a, b]$, we compute (for several values of $l$ )

$$
\mathcal{R}_{S}^{l}([a, b])=\log _{2}\left(\frac{\varrho_{6}^{l}}{\varrho_{7}^{l}}\right), \quad \varrho_{k}^{l}:=\sup \left\{\left|\left(\nabla^{l+1} f^{k}\right)_{i}\right|: x_{i}^{k} \in[a, b]\right\} .
$$

For our numerical testing process we consider $f_{i}^{0}=F\left(x_{i}^{0}\right), F(x)=e^{-2 x^{2}},\left(x_{i}^{0}\right)_{i}$ an $h_{0}$-uniform grid. In Table 3 we display the results corresponding to an initial mesh with $N=18$ points in $[-6,6]$, so that $x=0$ does not belong to the initial mesh, and in Table 4 we display the results obtained when a uniform mesh with $N=21$ points in $[-6,6]$, so that $x=0$ belongs to the initial grid, is used to compute $f^{0}$ (see also Fig. 10).

The tables show that, for these examples, the global regularity of the limit function is at least $r=1$. Moreover, when $x=0$ (the abscissa of the maximum of $F(x)$ ) is included in the initial grid, the global regularity of the limit function obtained with the Power $_{p}$ schemes is smaller than that obtained with the new schemes when $\max \{p, q\}>1$. According to Table 3 , in this case the limit function seems to be globally $C^{1}$ for $\max \{p, q\}>1$. In addition, we also observe in both tables that for $(p, q)=(2,2)$ we get the same smoothness as for the $S_{3,3}$ scheme, around $x=0$, much higher than that of the Power 2 scheme. 
Table 3 Regularity of $S^{\infty} f^{0}: \mathcal{R}_{S}^{l}([a, b])$ in Eq. 58 for $S=S_{3,3}, S_{H_{2}}$ and $\mathrm{SWH}_{p, q}$, for $3 \leq p+q \leq 4$

\begin{tabular}{lllllllllll}
\hline$l$ & $S_{3,3}$ & \multicolumn{3}{c}{$\mathcal{R}_{\mathrm{SWH}_{1,2}}^{l}$} & \multicolumn{3}{c}{$\mathcal{R}_{\mathrm{SWH}_{2,1}}^{l}$} & $\mathcal{R}_{\mathrm{SWH}_{2,2}}^{l}$ & $\mathcal{R}_{S_{H_{2}}}^{l}$ \\
\hline 0 & 0.95 & 1.00 & 1.00 & 1.00 & 0.96 & 1.00 & 0.95 & 1.00 & 0.94 & 1.00 \\
1 & 1.99 & 1.99 & 1.00 & 1.00 & 1.75 & 1.50 & 1.99 & 1.48 & 1.90 & 1.08 \\
2 & 2.81 & 2.84 & 1.00 & 1.00 & 1.64 & 1.01 & 2.84 & 1.00 & 2.06 & 1.08 \\
3 & 2.82 & 2.83 & 1.00 & 1.00 & 1.64 & 1.00 & 2.91 & 1.00 & 2.04 & 1.07 \\
4 & 2.83 & 2.83 & 1.00 & 1.00 & 1.64 & 1.00 & 2.85 & 1.00 & 1.78 & 1.07 \\
\hline
\end{tabular}

Left columns: $[a, b]=[-0.1,0.1]$. Right columns: $[a, b]=[-3,3]$. Initial data and limit functions displayed in Fig. 10, left column

\subsection{Approximation order}

The order of approximation of a subdivision scheme measures its ability to reconstruct smooth functions from relatively coarse samples. Given $f^{0}=\{F(n h)\}_{n \in \mathbb{Z}}$ where $F(x)$ is a smooth function, we study the difference between the limit function $f^{\infty}(x)=S^{\infty} f^{0}(x)$ and $F(x)$ in a given region by considering $S^{\infty} f^{0} \approx S^{L} f^{0}$ (with $L=7$ in all test cases) and measuring

$$
E_{S,[a, b]}(h):=\max \left\{\left|\left(S^{L} f^{0}\right)_{n}-F\left(n 2^{-L} h\right)\right|, n 2^{-L} h \in[a, b]\right\} \approx\left\|S^{\infty} f^{0}-F\right\|_{L^{\infty}([a, b])}
$$

In Tables 5-8 we display $E_{S,[a, b]}(h)$ for different values of $h$, different regions $[a, b]$ and different functions $F(x)$. The tables also show the numerical order of accuracy, $r_{n}$, obtained by a least squares fit of the data $\left(\log _{2}\left(h_{l}\right), \log _{2}\left(E_{S}\left(h_{l}\right)\right)\right.$ for $h_{l}=h_{0} / 2^{l}$, and a given value of $h_{0}$.

The ultimate purpose of the numerical testing process is twofold. On one hand the Tables show that, by choosing $p, q$ appropriately, it is possible to obtain the same order of approximation (as well as errors of a similar magnitude) as that of the $S_{3,3}$ scheme. In addition, the Tables also show that, for each scheme in the family, the order of approximation of the limit function is the same as the theoretical order of approximation after one iteration, $r_{t}$ in the Tables. For the new schemes proposed in the paper, $r_{t}$ is obtained in Corollary 12 for convex regions, but it can also be

Table 4 Same as Table 3. Initial data and limit functions displayed in Fig. 10, right column

\begin{tabular}{|c|c|c|c|c|c|c|c|c|c|c|}
\hline \multirow{2}{*}{$\frac{l}{0}$} & \multicolumn{2}{|l|}{$\mathcal{R}_{S_{3,3}}^{l}$} & \multicolumn{2}{|c|}{$\mathcal{R}_{\mathrm{SWH}_{1,2}}^{l}$} & \multicolumn{2}{|c|}{$\mathcal{R}_{\mathrm{SWH}_{2,1}}^{l}$} & \multicolumn{2}{|c|}{$\mathcal{R}_{\mathrm{SWH}_{2,2}}^{l}$} & \multicolumn{2}{|c|}{$\mathcal{R}_{S_{H_{2}}}^{l}$} \\
\hline & 0.91 & 1.00 & 1.00 & 1.00 & 1.00 & 1.00 & 0.95 & 1.00 & 1.00 & 1.00 \\
\hline 1 & 1.99 & 1.99 & 1.69 & 1.69 & 1.44 & 1.44 & 1.93 & 1.93 & 1.00 & 1.00 \\
\hline 2 & 2.82 & 2.82 & 1.63 & 1.63 & 1.48 & 1.48 & 2.47 & 1.34 & 1.00 & 1.00 \\
\hline 3 & 2.83 & 2.83 & 1.63 & 1.63 & 1.48 & 1.48 & 2.58 & 1.27 & 1.00 & 1.00 \\
\hline 4 & 2.83 & 2.83 & 1.38 & 1.38 & 1.47 & 1.47 & 2.64 & 1.30 & 1.00 & 1.00 \\
\hline
\end{tabular}



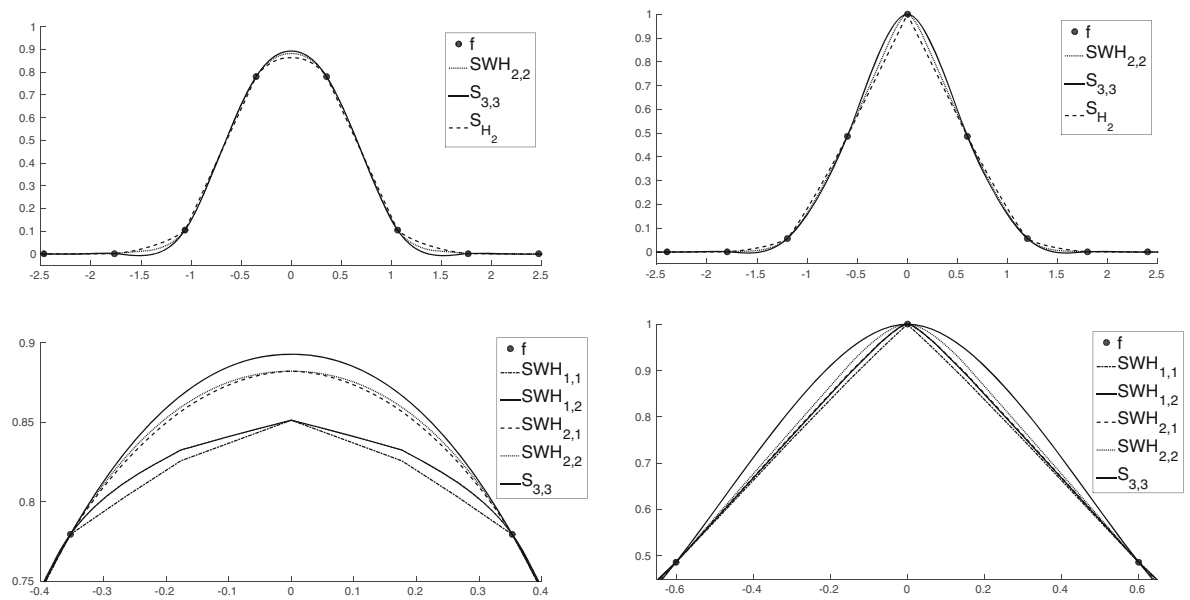

Fig. 10 Limit function from coarse Gaussian data. Initial data marked with •

obtained by Taylor expansions in regions where $\mathcal{L}_{l, r} \circ \nabla^{2}$ does not change sign. For the purpose of illustration, we include the result of the algebraic computation (done with Mathematica) for $p=2, q=2$,

$\left(\mathrm{SWH}_{p, q} f\right)_{2 j+1}=F((2 n+1) h / 2)+\frac{h^{6}}{2048}\left(\frac{15 F^{I V}((2 j+1) h / 2)^{2}}{F^{\prime \prime}((2 j+1) h / 2)}+10 F^{V I}((2 j+1) h / 2)\right)$,

which holds for smooth functions, provided that $\left(\mathcal{L}_{l, r} \circ \nabla^{2} f\right)_{2 j+1}$ have the same sign. We notice further that if $F$ is a smooth function and $f_{j}=F\left(x_{j}\right)$, straightforward Taylor expansions lead to

$$
\begin{aligned}
& \left(\mathcal{L}_{2,2} \nabla^{2} f\right)_{2 n+2 m+1}=2 F^{\prime \prime}\left(x_{n}\right) h^{2}+(1+2 m) F^{\prime \prime \prime}\left(x_{n}\right) h^{3}+\left(\frac{2}{3}+m+m^{2}\right) F^{I V}\left(x_{n}\right) h^{4}+O\left(h^{5}\right), \\
& \left(\mathcal{L}_{3,1} \nabla^{2} f\right)_{2 n+2 m+1}=2 F^{\prime \prime}\left(x_{n}\right) h^{2}+(1+2 m) F^{\prime \prime \prime}\left(x_{n}\right) h^{3}+\left(-\frac{1}{3}+m+m^{2}\right) F^{I V}\left(x_{n}\right) h^{4}+O\left(h^{5}\right), \\
& \left(\mathcal{L}_{1,3} \nabla^{2} f\right)_{2 n+2 m+1}=2 F^{\prime \prime}\left(x_{n}\right) h^{2}+(1+2 m) F^{\prime \prime \prime}\left(x_{n}\right) h^{3}+\left(-\frac{1}{3}+m+m^{2}\right) F^{I V}\left(x_{n}\right) h^{4}+O\left(h^{5}\right) .
\end{aligned}
$$

Table 5 Approximation order for Gaussian data: $E_{S,[a, b]}\left(h_{l}\right),[a, b]=[-0.4,0.4], h_{l}=2^{-l} h_{0}$, and several schemes

\begin{tabular}{lllllllll}
\hline$l$ & $S_{3,3}$ & $S_{H_{2}}$ & $S_{H_{3}}$ & $\mathrm{SWH}_{1,1}$ & $\mathrm{SWH}_{2,1}$ & $\mathrm{SWH}_{2,2}$ & $\mathrm{SWH}_{3,1}$ & $\mathrm{SWH}_{3,2}$ \\
\hline 0 & $4.3 \mathrm{e}-6$ & $2.4 \mathrm{e}-4$ & $1.1 \mathrm{e}-4$ & $1.7 \mathrm{e}-4$ & $1.7 \mathrm{e}-5$ & $6.3 \mathrm{e}-6$ & $1.6 \mathrm{e}-5$ & $3.5 \mathrm{e}-6$ \\
1 & $7.2 \mathrm{e}-8$ & $1.8 \mathrm{e}-5$ & $7.0 \mathrm{e}-6$ & $1.1 \mathrm{e}-5$ & $5.4 \mathrm{e}-7$ & $1.0 \mathrm{e}-7$ & $5.3 \mathrm{e}-7$ & $5.7 \mathrm{e}-8$ \\
2 & $1.1 \mathrm{e}-9$ & $1.2 \mathrm{e}-6$ & $4.4 \mathrm{e}-7$ & $7.5 \mathrm{e}-7$ & $1.7 \mathrm{e}-8$ & $1.7 \mathrm{e}-9$ & $1.6 \mathrm{e}-8$ & $9.0 \mathrm{e}-10$ \\
3 & $1.8 \mathrm{e}-11$ & $8.0 \mathrm{e}-8$ & $2.7 \mathrm{e}-8$ & $4.7 \mathrm{e}-8$ & $5.3 \mathrm{e}-10$ & $2.7 \mathrm{e}-11$ & $5.3 \mathrm{e}-10$ & $1.4 \mathrm{e}-11$ \\
$r_{n}$ & 5.96 & 3.85 & 3.98 & 3.95 & 4.99 & 5.94 & 4.98 & 5.97 \\
$r_{t}$ & 6 & 4 & 4 & 4 & 5 & 6 & 5 & 6 \\
\hline
\end{tabular}


Hence, at smooth convex regions (at least for $h$ small enough) $\mathcal{L}_{l, r} \circ \nabla^{2}$ does not change sign. Moreover, if $x_{n}=n h$ is an inflection point and $F^{\prime \prime \prime}\left(x_{n}\right) \neq 0$, the formulas above show that, for $h$ small enough and $(l, r)=(1,3),(3,1),(2,2)$, $\left(\mathcal{L}_{l, r} \nabla^{2} f\right)_{2 n+m}$ do not change sign for $m \geq 0$ or $m<0$, and the calculation of $r_{t}$ by Taylor expansions is feasible. This is the case of the two functions considered in the numerical tests.

The testing process below is carried out for the family of schemes $\mathrm{SWH}_{p, q}$. We have also performed the same study for the $\mathrm{SHW}_{q, p}$ family. As expected, the resulting tables are similar and the conclusions are also the same, hence we omit them. We include the errors corresponding to the $S_{3,3}$ and $S_{H_{p}}$ schemes for the sake of comparison. An $*$ in the Table 6 means that it is not possible to find $r_{t}$ by Taylor expansions.

\subsubsection{Gaussian data}

We consider $F(x)=e^{-2 x^{2}}, h_{0}=0.1$. Table 5 displays the results for the region $[a, b]=[-0.4,0.4]$, where $F$ is convex and $\left|F^{\prime \prime}(x)\right| \geq\left|F^{\prime \prime}(0.4)\right| \approx 1.04$. We observe that the estimated order of approximation of $\mathrm{SWH}_{p, q}$ coincides with $r_{t}$, the order of approximation after one iteration in the convex region.

In Table 6 we display the corresponding results for $[a, b]=[-1,-0.3]$, which contains the inflection point $x=0.5$. The Table indicates that the computed $r_{n}$ coincides with $r_{t}$ for all the nonlinear 6-point schemes. As observed above, $r_{t}$ can still be computed by Taylor expansions for the 6-points nonlinear schemes. It should be noticed that for $F(x)=e^{-2 x^{2}}$

$$
\frac{F^{I V}(x)^{2}}{F^{\prime \prime}(x)}=64 e^{-2 x^{2}} \frac{\left(16 x^{4}-24 x^{2}+3\right)^{2}}{4 x^{2}-1}
$$

which has a vertical asymptote in $x= \pm 0.5$ and hence it is unbounded around the inflection points. Since $F^{\prime \prime}((2 n+1) h / 2)=F^{\prime \prime \prime}(n h) h / 2+O\left(h^{2}\right)$, Eq. 60 leads to

$$
\left(\mathrm{SWH}_{p, q} d d f\right)_{2 n+1}=F((2 n+1) h / 2)+O\left(h^{5}\right),
$$

around the inflection point, i.e., the order of approximation after one iteration is 5 in the non-convex region. Performing the analogous computation for $(p, q)=(3,1)$,

Table 6 Same as Table 5 for $[a, b]=[-1,-0.3]$

\begin{tabular}{lllllllll}
\hline$l$ & $S_{3,3}$ & $S_{H_{2}}$ & $S_{H_{3}}$ & $\mathrm{SWH}_{1,1}$ & $\mathrm{SWH}_{2,1}$ & $\mathrm{SWH}_{2,2}$ & $\mathrm{SWH}_{3,1}$ & $\mathrm{SWH}_{3,2}$ \\
\hline 0 & $3.1 \mathrm{e}-6$ & $6.1 \mathrm{e}-4$ & $5.9 \mathrm{e}-4$ & $1.0 \mathrm{e}-4$ & $1.5 \mathrm{e}-5$ & $8.9 \mathrm{e}-6$ & $1.5 \mathrm{e}-5$ & $3.3 \mathrm{e}-6$ \\
1 & $5.1 \mathrm{e}-8$ & $7.7 \mathrm{e}-5$ & $7.6 \mathrm{e}-5$ & $7.3 \mathrm{e}-6$ & $5.3 \mathrm{e}-7$ & $2.1 \mathrm{e}-7$ & $5.3 \mathrm{e}-7$ & $5.0 \mathrm{e}-8$ \\
2 & $8.1 \mathrm{e}-10$ & $9.7 \mathrm{e}-6$ & $9.6 \mathrm{e}-6$ & $4.6 \mathrm{e}-7$ & $1.7 \mathrm{e}-8$ & $5.7 \mathrm{e}-9$ & $1.6 \mathrm{e}-8$ & $7.4 \mathrm{e}-10$ \\
3 & $1.3 \mathrm{e}-11$ & $1.2 \mathrm{e}-6$ & $1.2 \mathrm{e}-6$ & $2.9 \mathrm{e}-8$ & $5.3 \mathrm{e}-10$ & $1.5 \mathrm{e}-10$ & $5.3 \mathrm{e}-10$ & $1.1 \mathrm{e}-11$ \\
$r_{n}$ & 5.97 & 2.99 & 2.98 & 3.95 & 4.95 & 5.26 & 4.95 & 6.07 \\
$r_{t}$ & 6 & $*$ & $*$ & 4 & 5 & 5 & 5 & 6 \\
\hline
\end{tabular}


Table 7 Approximation order for Tangent data: $E_{S,[a, b]}\left(h_{l}\right),[a, b]=[0.1,0.3], h_{l}=2^{-l} h_{0}$ and several schemes

\begin{tabular}{lllllllll}
\hline$l$ & $S_{3,3}$ & $S_{H_{2}}$ & $S_{H_{3}}$ & $\mathrm{SWH}_{1,1}$ & $\mathrm{SWH}_{2,1}$ & $\mathrm{SWH}_{2,2}$ & $\mathrm{SWH}_{3,1}$ & $\mathrm{SWH}_{3,2}$ \\
\hline 2 & $1.6 \mathrm{e}-5$ & $7.3 \mathrm{e}-6$ & $1.4 \mathrm{e}-4$ & $3.3 \mathrm{e}-4$ & $9.0 \mathrm{e}-5$ & $2.8 \mathrm{e}-5$ & $1.8 \mathrm{e}-5$ & $1.7 \mathrm{e}-5$ \\
3 & $2.8 \mathrm{e}-7$ & $4.8 \mathrm{e}-7$ & $1.1 \mathrm{e}-5$ & $2.0 \mathrm{e}-5$ & $2.3 \mathrm{e}-6$ & $4.5 \mathrm{e}-7$ & $2.1 \mathrm{e}-6$ & $2.8 \mathrm{e}-7$ \\
4 & $4.7 \mathrm{e}-9$ & $3.1 \mathrm{e}-8$ & $7.5 \mathrm{e}-7$ & $1.3 \mathrm{e}-6$ & $6.9 \mathrm{e}-8$ & $7.6 \mathrm{e}-9$ & $6.6 \mathrm{e}-8$ & $4.7 \mathrm{e}-9$ \\
5 & $7.7 \mathrm{e}-11$ & $1.9 \mathrm{e}-9$ & $5.0 \mathrm{e}-8$ & $1.3 \mathrm{e}-7$ & $2.1 \mathrm{e}-9$ & $1.2 \mathrm{e}-10$ & $2.0 \mathrm{e}-9$ & $7.7 \mathrm{e}-11$ \\
$r_{n}$ & 5.88 & 3.96 & 3.80 & 4.09 & 5.11 & 5.94 & 5.02 & 5.93 \\
\hline
\end{tabular}

$(3,2)$ we find that the 'theoretical' approximation order after one iteration is 5 and 6 , respectively, also in the non-convex region.

We also remark that, in all the tables displayed, the magnitude of the errors corresponding to the 6-point nonlinear schemes whose order of accuracy is 4, 5 or 6 are similar to those of $S_{3,3}$ and better than that of the Power $p$ schemes.

\subsubsection{Tangent data}

We repeat the previous study for $F(x)=\tan (\pi x)$. In this case, the function is convex in the interval $[0.1,0.3]$, and changes convexity at $[-0.25,0.25]$. We consider $h_{0}=0.1$. The conclusions are similar. In particular, the magnitude of the errors is similar to those of $S_{3,3}$, and better than those obtained with the Power $_{p}$ schemes, for $\mathrm{SWH}_{p, q}$ schemes whose order of accuracy is 4,5 or 6 . We also remark that order of approximation of the scheme coincides with $r_{t}$ (not displayed in the tables). Notice that for $F(x)=\tan (\pi x)$

$$
F^{I V}(x) / F^{\prime \prime}(x)=8 \pi^{6}(\cos (2 \pi x)-5)^{2} \tan (\pi x) \sec ^{6}(\pi x)
$$

which is a bounded function around $x=0$. Hence, according to Eq. 60, the order of approximation after one iteration of $\mathrm{SWH}_{p, q} d d$ is 6 also in the non-convex region.

Table 8 Same as Table 7 for $[a, b]=[-0.25,0.25]$

\begin{tabular}{lllllllll}
\hline$l$ & $S_{3,3}$ & $S_{H_{2}}$ & $S_{H_{3}}$ & $\mathrm{SWH}_{1,1}$ & $\mathrm{SWH}_{2,1}$ & $\mathrm{SWH}_{2,2}$ & $\mathrm{SWH}_{3,1}$ & $\mathrm{SWH}_{3,2}$ \\
\hline 2 & $3.5 \mathrm{e}-6$ & $6.2 \mathrm{e}-5$ & $6.2 \mathrm{e}-5$ & $2.1 \mathrm{e}-4$ & $2.2 \mathrm{e}-5$ & $6.1 \mathrm{e}-6$ & $7.1 \mathrm{e}-5$ & $3.7 \mathrm{e}-6$ \\
3 & $6.0 \mathrm{e}-8$ & $7.8 \mathrm{e}-6$ & $7.8 \mathrm{e}-6$ & $1.2 \mathrm{e}-5$ & $6.1 \mathrm{e}-7$ & $9.9 \mathrm{e}-8$ & $5.6 \mathrm{e}-7$ & $6.1 \mathrm{e}-8$ \\
4 & $1.0 \mathrm{e}-9$ & $9.7 \mathrm{e}-7$ & $9.7 \mathrm{e}-7$ & $7.2 \mathrm{e}-7$ & $1.8 \mathrm{e}-8$ & $1.6 \mathrm{e}-9$ & $1.8 \mathrm{e}-8$ & $1.0 \mathrm{e}-9$ \\
5 & $1.6 \mathrm{e}-11$ & $1.2 \mathrm{e}-7$ & $1.2 \mathrm{e}-7$ & $4.4 \mathrm{e}-8$ & $5.6 \mathrm{e}-10$ & $2.6 \mathrm{e}-11$ & $5.5 \mathrm{e}-10$ & $1.6 \mathrm{e}-11$ \\
$r_{n}$ & 5.90 & 3.00 & 3.00 & 4.07 & 5.08 & 5.93 & 5.01 & 5.93 \\
\hline
\end{tabular}




\section{Conclusions}

We have constructed two families of non-oscillatory subdivision schemes that can be considered nonlinear versions of the 6-point Deslauries-Dubuc interpolatory subdivision scheme. We have studied their convergence by exploiting the (piecewise) smoothness properties of the functions that define these subdivision schemes, following a novel technique developed in [19]. The stability of these schemes turns out to be more difficult to study with the techniques employed in [22] and we have explored this issue computationally. The numerical results reveal indeed that the techniques based on finding appropriate bounds for the Generalized Jacobian of the second difference scheme, as in [22], have no chance to succeed, except for $(p, q)=(1,1)$ (where contractivity might be proven for $L=2)$ and $(p, q)=(2,1)$ (for $L=4)$.

We have also performed several numerical experiments that suggest that the approximation properties of the new schemes can be as good as those of the 6-point linear scheme when reconstructing smooth functions. In addition, numerical experiments show that the smoothness of the limit functions obtained from convex data may be larger than the smoothness of the limit functions obtained with Power $_{p}$ schemes.

Acknowledgments The authors acknowledge support from Project MTM2014-54388 (MINECO, Spain) and the FPU14/02216 grant (MECD, Spain).

\section{Appendix A. Generalized Gradients and Generalized Jacobians}

The schemes considered in this paper involve nonlinear functions that are continuous but only piecewise differentiable. As shown in [22], the theory developed by Oswald and Harizanov in [19] can be used to analyze the stability of such schemes, provided that the functions that define them belong to a special class of piecewise smooth functions, for which uniformly bounded Generalized Gradients can be defined. For the sake of completeness, and ease of future reference, we include here the relevant theoretical results, including proofs, in a framework broad enough to cover the schemes considered in $[19,22]$ and in this paper. We also remark that the theory of Generalized Gradients has been developed in greater generality by Clarke in a series of papers (see [7, 21]).

\section{The $C_{p w}^{1}\left(\mathbb{R}^{m}\right)$ class of functions: Generalized Gradients}

The $C_{p w}^{1}\left(\mathbb{R}^{m}\right)$ class of functions was defined in [19]. Functions $\psi: \mathbb{R}^{m} \rightarrow \mathbb{R}$ in this class are continuous, piecewise smooth and have directional derivatives except (maybe) at $0 \in \mathbb{R}^{m}$ and across certain hyperplanes separating regions of $C^{1}$ smoothness. Directional derivatives along the separating hyperplanes do, also, exist.

We shall assume that there exist a finite number of hyperplanes $\left\{\mathcal{H}_{i}\right\}_{i}, 0 \in \mathcal{H}_{i}$, such that $\psi$ is continuously differentiable $\forall x \in \mathbb{R}^{m} \backslash \bigcup_{i} \mathcal{H}_{i}$ and $\left.\psi\right|_{\mathcal{H}_{i}}$ is continuously differentiable (as a function of $m-1$ variables) except maybe at $x=0$. In this context $\mathbb{R}^{m} \backslash \bigcup_{i} \mathcal{H}_{i}$ is the union of a finite number of disjoint open convex sectors in $\mathbb{R}^{m}$, 
which we shall denote by $\Omega_{j}$. Therefore $\mathbb{R}^{m}=\left(\bigcup_{j} \Omega_{j}\right) \cup\left(\bigcup_{i} \mathcal{H}_{i}\right)$, the sets $\bigcup_{j} \Omega_{j}$ and $\bigcup_{i} \mathcal{H}_{i}$ are disjoint, and $\partial \Omega_{j}$, the boundary of $\Omega_{j}$, is always included in the union of the separating hyperplanes.

In addition, functions in $C_{p w}^{1}\left(\mathbb{R}^{m}\right)$ have uniformly bounded gradients in smooth regions, i.e.

$$
C_{\|\cdot\|}:=\sup _{j} \sup _{p \in \Omega_{j}}\|\nabla \psi(p)\|<\infty,
$$

and the smooth gradients satisfy the following compatibility condition on the separating hyperplanes: Let $0 \neq x \in \partial \Omega \subset \mathcal{H}$, where $\Omega$ is a smoothness region for $\psi$ and $\mathcal{H}$ is one of the hyperplanes separating the regions of smoothness of $\psi$. Then

$$
\left(\lim _{x \leftarrow p \in \Omega} \nabla \psi(p)\right) \cdot w=D_{w} \psi(x), \quad \forall 0 \neq w \in \mathcal{H}
$$

where $D_{w} \psi(x)$ is the derivative of $\psi$ at $x$ in the direction of $w$.

Functions in $C_{p w}^{1}\left(\mathbb{R}^{m}\right)$ admit Generalized Gradients. As the standard gradient of a smooth function, the main property of a Generalized Gradient is that the associated linear map recovers all directional derivatives that 'make sense' at a given point. Conditions (A.1)-(A.2) ensure that

$$
D \psi(x):=\left\{\begin{array}{ll}
\nabla \psi(x) & \text { if } x \in \Omega \text { (smoothness region) } \\
\lim _{x \leftarrow p \in \Omega} \nabla \psi(p) & \text { if } x \in \partial \Omega
\end{array} \quad \forall 0 \neq x \in \mathbb{R}^{m}, \quad D \psi(0)=0\right.
$$

provides an adequate definition of a Generalized Gradient of $\psi \in C_{p w}^{1}\left(\mathbb{R}^{m}\right)$, since for each $0 \neq x \in \mathbb{R}^{m}, D \psi(x)$ defines a linear map that satisfies

$$
D_{v} \psi(x)=D \psi(x) \cdot v
$$

for any $\overrightarrow{0} \neq v \in \mathbb{R}^{m}$ when $x$ belongs to a smoothness region, and also for any $0 \neq v \in \mathcal{H}$ when $0 \neq x \in \mathcal{H}$.

Notice that $D \psi(x)$ in Eq. A.3 might not be uniquely defined when $x$ belongs to a hyperplane separating two or more regions of smoothness, if the limit in Eq. A.3 is different for different smoothness regions with a common boundary. However the compatibility condition (A.2) ensures (A.4) for all directional derivatives that make sense, independently of the chosen definition for the vector $D \psi(x)$.

The following results generalize some of the properties satisfied by the gradient of a smooth function.

Lemma 18 (Generalized MVT) Let $\psi \in \mathbb{C}_{p w}^{1}\left(\mathbb{R}^{m}\right), x, y \in \mathbb{R}^{m}, x \neq y$ and $\Gamma:=$ $\{t x+(1-t) y, \quad t \in(0,1)\}$. Then, if $\Gamma \subset \Omega$ (smoothness region for $\psi)$ or $\Gamma \subset$ $\mathcal{H}-\{0\}$, then $\exists \hat{t} \in(0,1)$ such that

$$
\psi(x)-\psi(y)=D \psi(\xi)(x-y), \quad \xi=\hat{t} x+(1-\hat{t}) y,
$$

where $D \psi$ is a generalized gradient of $\psi$.

Proof Define $\gamma:[0,1] \rightarrow \mathbb{R}^{m}, g:[0,1] \rightarrow \mathbb{R}$

$$
\gamma(t):=t x+(1-t) y=y+t(x-y), \quad g(t):=\psi(\gamma(t)) .
$$


Then,

$$
g(t+h)-g(t)=\psi(\gamma(t)+h(x-y))-\psi(\gamma(t)) \quad \forall h \in \mathbb{R}
$$

hence, under the hypothesis of the Lemma, $\gamma((0,1))=: \Gamma \subset \Omega$ or $\Gamma \subset \mathcal{H}-\{0\}$, and

$$
g^{\prime}(t)=\lim _{h \rightarrow 0} \frac{g(t+h)-g(t)}{h}=D_{x-y} \psi(\gamma(t))=D \psi(\gamma(t))(x-y)
$$

for any Generalized Gradient $D \psi$ and for any $t \in(0,1)$ (notice that if $x, y \in \mathcal{H}$, a separating hyperplane, then $x-y \in \mathcal{H})$. Since $g(t)$ is differentiable in $(0,1)$, by the classical Mean Value Theorem (MTV)

$$
\exists \hat{t} \in(0,1): \quad \psi(x)-\psi(y)=g(1)-g(0)=g^{\prime}(\hat{t})=D \psi(\xi)(x-y),
$$

with $\xi=\gamma(\hat{t})=\hat{t} x+(1-\hat{t}) y$.

Corollary 19 Let $\psi \in C_{p w}^{1}\left(\mathbb{R}^{m}\right)$. Then $\forall x, y \in \mathbb{R}^{m}$

$$
|\psi(x)-\psi(y)| \leq C_{1}|| x-y\left\|\quad C_{1}=\sup _{j} \sup _{p \in \Omega_{j}}\right\| \nabla \psi(p) \|_{1}
$$

Proof We consider again the straight line $\gamma(t)=y+t(x-y)$ and the function $g(t)=\psi(\gamma(t))$ in Eq. A.5. Notice that $\gamma(t)$ can either cut the separating hyperplanes at a finite number of points, or belong entirely to one of them. We prove (A.9) in each case.

Let us assume that that $0 \leq t_{1}<\ldots<t_{k} \leq 1$ are such that $\gamma\left(t_{k}\right)$ are the cutting points with the (finite number of) hyperplanes separating the smoothness regions of $\psi$. We consider $t_{0}=0$ and $t_{k+1}=1$. Without loss of generality, we may assume $t_{0}<t_{1}<\ldots<t_{k+1}$, hence $\Gamma_{l}:=\left\{\gamma(t), t \in\left(t_{l}, t_{l+1}\right)\right\}$ is included in one of the smoothness regions of $\psi$, for $l=0, \cdots, k$. By the previous lemma,

$g\left(t_{l+1}\right)-g\left(t_{l}\right)=g^{\prime}\left(\hat{t}_{l}\right)=D \psi\left(\gamma\left(\hat{t}_{l}\right)\right)\left(\gamma\left(t_{l+1}\right)-\gamma\left(t_{l}\right)\right)=D \psi\left(\gamma\left(\hat{t}_{l}\right)\right)\left(t_{l+1}-t_{l}\right)(x-y), \quad l=0, \ldots, k$.

Thus, using Lemma 18 and considering the Generalized Gradient $D \psi$ in Eq. A.3, we can write

$$
\begin{aligned}
|\psi(x)-\psi(y)| & =\left|\sum_{l=0}^{k} g\left(t_{l+1}\right)-g\left(t_{l}\right)\right|=\left|\sum_{l=0}^{k} D \psi\left(\hat{t}_{l}\right)\left(t_{l+1}-t_{l}\right)(x-y)\right| \leq \sum_{l=0}^{k}\left(t_{l+1}-t_{l}\right)\left|D \psi\left(\hat{t}_{l}\right)(x-y)\right| \\
& \leq \sum_{l=0}^{k}\left(t_{l+1}-t_{l}\right)|| D \psi\left(\hat{t}_{l}\right)\left\|_{1}|| x-y\right\|_{\infty} \leq C_{1}|| x-y\left\|_{\infty} \sum_{l=0}^{k}\left(t_{l+1}-t_{l}\right)=C_{1}|| x-y\right\|_{\infty} .
\end{aligned}
$$

since (A.1) leads to $\|D \psi(x)\|_{1} \leq C_{1}, \forall x \in \mathbb{R}^{m}$, for $D \psi$ in Eq. A.3.

Let us assume now that $\{\gamma(t), t \in \mathbb{R}\} \subset \mathcal{H}$. Then, either $\Gamma \in \mathcal{H}-\{0\}$ or $\exists \bar{t} \in(0,1)$ such that $\gamma(\bar{t})=0$. In both cases, the result follows easily from Lemma 18, using the same arguments as before. 
The following result establishes that the chain rule holds for the composition of $C_{p w}^{1}\left(\mathbb{R}^{m}\right)$ functions with Lipschitz curves.

Theorem 20 Let $\gamma:[a, b] \rightarrow \mathbb{R}^{m}$ be a Lipschitz curve, $\psi: \mathbb{R}^{m} \rightarrow \mathbb{R}$ a function in $C_{p w}^{1}\left(\mathbb{R}^{m}\right)$ and $D \psi$ a generalized gradient of $\psi$. Then $\tilde{\gamma}=\psi \circ \gamma:[a, b] \rightarrow \mathbb{R}$ is also a Lipschitz curve, and

$$
\tilde{\gamma}^{\prime}(t)=D \psi(\gamma(t)) \gamma^{\prime}(t) \quad \text { a.e. in }(a, b) .
$$

Proof The curve $\tilde{\gamma}$ is Lipschitz because both $\gamma$ and $\psi$ are Lipschitz. Let $A_{\gamma}$ be the set of points where $\gamma$ is not differentiable and $A_{\tilde{\gamma}}$ the corresponding set for $\tilde{\gamma}$. Notice that both sets have zero measure, since Lipschitz functions are a.e. differentiable.

Since $\mathbb{R}^{m}=\left(\bigcup_{i} \mathcal{H}_{i}\right) \cup\left(\bigcup_{j} \Omega_{j}\right)$ it follows that $[a, b]=\left(\bigcup_{i} \tilde{\mathcal{H}}_{i}\right) \cup\left(\bigcup_{j} \tilde{\Omega}_{j}\right) \cup O$, where

$\tilde{\mathcal{H}}_{i}:=\left\{t \in[a, b], 0 \neq \gamma(t) \in \mathcal{H}_{i}\right\}, \quad \tilde{\Omega}_{j}:=\left\{t \in[a, b]: \gamma(t) \in \Omega_{j}\right\}, \quad O=\{t \in[a, b]: \gamma(t)=0\}$.

Let us denote by $E_{i}$ the set of isolated points in $\tilde{\mathcal{H}}_{i}$ and $F$ the set of isolated points in $O$. These sets are countable ${ }^{3}$, hence $\left(\bigcup_{i} E_{i}\right) \cup F$ is also countable. Therefore $B=A_{\gamma} \cup A_{\tilde{\gamma}} \cup\left(\bigcup_{i} E_{i}\right) \cup F$ is also a set of zero-measure. We shall check that (A.10) holds $\forall t \in(a, b) \backslash B$. Notice that $\forall t \in(a, b) \backslash B$ both $\tilde{\gamma}^{\prime}(t)$ and $\gamma^{\prime}(t)$ exist, since $t \notin A_{\tilde{\gamma}} \cap A_{\gamma}$, and can be computed as

$$
\tilde{\gamma}^{\prime}(t)=\lim _{n \rightarrow \infty} \frac{\tilde{\gamma}\left(t_{n}\right)-\tilde{\gamma}(t)}{t_{n}-t}, \quad \gamma^{\prime}(t)=\lim _{n \rightarrow \infty} \frac{\tilde{\gamma}\left(t_{n}\right)-\tilde{\gamma}(t)}{t_{n}-t}
$$

for any sequence $\left\{t_{n}\right\}_{n}$ such that $t_{n} \rightarrow t$.

Let us assume that $t \in \tilde{\Omega}_{j} \backslash B$ and let $\left\{t_{n}\right\}$ be a sequence such that $t_{n} \rightarrow t$. Since $\gamma$ is continuous, $\gamma\left(t_{n}\right) \rightarrow t$. Moreover, $\gamma\left(t_{n}\right) \in \Omega_{j}$ for $n$ large enough, because $\Omega_{j}$ is an open set. Since $\Omega_{j}$ is convex, $\Gamma_{n} \subset \Omega_{j}$, where $\Gamma_{n}$ is the segment joining $\gamma\left(t_{n}\right)$ and $\gamma(t)$. Hence, for $n$ large enough, using Lemma 18 we can write

$$
\tilde{\gamma}\left(t_{n}\right)-\tilde{\gamma}(t)=D \psi\left(\xi_{n}\right)\left(\gamma\left(t_{n}\right)-\gamma(t)\right), \quad \xi_{n} \in \Gamma_{n} .
$$

Since $\gamma\left(t_{n}\right) \rightarrow \gamma(t)$, we have that $\xi_{n} \rightarrow \gamma(t)$. In smooth regions, any generalized gradient must be uniquely defined as $D \psi(x)=\nabla \psi(x)$. Since $\left.\psi\right|_{\Omega_{j}} \in C^{1}\left(\Omega_{j}\right)$, we have that

Hence,

$$
\lim _{n \rightarrow \infty} D \psi\left(\xi_{n}\right)=D \psi(\gamma(t))
$$

$$
\tilde{\gamma}^{\prime}(t)=\lim _{n \rightarrow \infty} \frac{\tilde{\gamma}\left(t_{n}\right)-\tilde{\gamma}(t)}{t_{n}-t}=\lim _{n \rightarrow \infty} D \psi\left(\xi_{n}\right) \frac{\gamma\left(t_{n}\right)-\gamma(t)}{t_{n}-t}=D \psi(\gamma(t)) \gamma^{\prime}(t) .
$$

Let us assume now that $t \in \tilde{\mathcal{H}}_{i} \backslash B$, with $\mathcal{H}_{i}$ one of the separating hyperplanes. Since $t \notin E_{i}, \exists t_{n} \rightarrow t, t_{n} \in \tilde{\mathcal{H}}_{i}$. Since $\gamma(t)$ is continuous and $\gamma(t) \neq 0$, we can also

\footnotetext{
${ }^{3}$ If $x \in E$ is an isolated point, $\exists V_{x}$ open, such that $V_{x} \cap E=\{x\}$, and $\mathbb{R}$ is a second-countable space.
} 
assume that $\Gamma_{n} \subset \mathcal{H}_{i} \backslash\{0\}$ and, by Lemma 18, we get (A.12). Notice that Eq. A.13 also holds, because of the requirement that $\psi_{\left.\right|_{\mathcal{H}}}$ is continuously differentiable (as a function of $\mathbb{R}^{m-1}$ variables) in $\mathcal{H} \backslash\{0\}$. Hence, Eq. A.14 also follows in this case.

Finally, let us assume that $t \in O \backslash B$. Since $t \in O \backslash F, \exists t_{n} \rightarrow t, \gamma\left(t_{n}\right)=0$. Then

$$
\tilde{\gamma}^{\prime}(t)=\lim _{n \rightarrow \infty} \frac{\tilde{\gamma}\left(t_{n}\right)-\tilde{\gamma}(t)}{t_{n}-t}=0=D \psi(\gamma(t)) \lim _{n \rightarrow \infty} \frac{\gamma\left(t_{n}\right)-\gamma(t)}{t_{n}-t}
$$

since $\tilde{\gamma}\left(t_{n}\right)=\tilde{\gamma}(t)$ and $\gamma\left(t_{n}\right)=\gamma(t)$.

The chain rule (A.10) turns out to be the key property for our purposes. The next result shows that this property is also satisfied by a possibly wider class of functions.

Corollary 21 Let $\psi \in C^{1}\left(\mathbb{R}^{m}\right), M: \mathbb{R}^{p} \rightarrow \mathbb{R}^{m}$ a linear map and $\gamma:[a, b] \rightarrow \mathbb{R}^{p}$ a Lipschitz curve. Then the curve $\tilde{\gamma}:=\psi \circ M \circ \gamma$ is also Lipschitz and satisfies

$$
\tilde{\gamma}^{\prime}(t)=D \psi(M \gamma(t)) M \gamma^{\prime}(t) \quad \text { a.e.in }(a, b)
$$

Proof Note that $\gamma_{M}:=M \circ \gamma:[a, b] \rightarrow \mathbb{R}^{m}$ is continuous and $\gamma_{M}^{\prime}(t)=M \gamma^{\prime}(t)$ a.e in $(a, b)$. Hence, the result follows from applying Theorem 20 to $\psi$ and $\gamma_{M}$.

This result allows us to write $D(\psi \circ M)(x)=D \psi(M x) M$ as a Generalized Gradient of $\psi \circ M$ when $\psi \in C_{p w}^{1}\left(\mathbb{R}^{m}\right)$. Notice that $\|D(\psi \circ M)(x)\|$ is also uniformly bounded. Hence, we may also associate the notion of Generalized Gradients to certain functions, related (by composition) to $C_{p w}^{1}\left(\mathbb{R}^{m}\right)$ functions.

\section{Generalized Jacobians}

The notion of Generalized Gradient leads, in a rather natural way, to that of Generalized Jacobian for functions $\psi: \mathbb{R}^{m} \rightarrow \mathbb{R}^{p}, \psi=\left(\psi_{1}, \ldots, \psi_{p}\right), \psi_{i} \in \mathbb{C}_{p w}^{1}\left(\mathbb{R}^{m}\right)$. As in the smooth case,

$$
D \psi(x):=\left(\begin{array}{c}
D \psi_{1}(x) \\
\vdots \\
D \psi_{p}(x)
\end{array}\right)
$$

provides the definition of a Generalized Jacobian of $\psi$ at $x \in \mathbb{R}^{m}$. Obviously, the definition may not be unique, but, as stated below, the linear maps associated to such matrices also satisfy the chain rule for the composition with Lipschitz curves.

Theorem 22 Let $\gamma:[a, b] \rightarrow \mathbb{R}^{m}$ be a Lipschitz curve, $\psi: \mathbb{R}^{m} \rightarrow \mathbb{R}^{p}, \psi=$ $\left(\psi_{1}, \cdots, \psi_{p}\right)$, a function such that $\psi_{i} \in C_{p w}^{1}\left(\mathbb{R}^{m}\right)$ and $D \psi$ a generalized Jacobian of $\psi$. Then $\tilde{\gamma}=\psi \circ \gamma$ is also Lipschitz and

$$
\tilde{\gamma}^{\prime}(t)=D \psi(\gamma(t)) \gamma^{\prime}(t), \quad \text { a.e. in }(a, b) .
$$


Proof Since $\tilde{\gamma}_{i}=\psi_{i} \circ \gamma$, it is Lipschitz and Eq. A.15 holds a.e. for each $1 \leq i \leq p$. Hence

$\tilde{\gamma}_{i}^{\prime}(t)=D \psi_{i}(\gamma(t)) \gamma^{\prime}(t), \quad \forall t \in[a, b] \backslash I_{i} \quad \rightarrow \quad \tilde{\gamma}^{\prime}(t)=D \psi(\gamma(t)) \gamma^{\prime}(t), \quad \forall t \in[a, b] \backslash \bigcup_{i} I_{i}$

since $\bigcup_{i} I_{i}$ is a null set. Hence the chain rule is valid a.e. in $(a, b)$.

In [19], the authors extend the notion of Generalized Jacobian to any scheme $S$ that is defined by functions in $C_{p w}^{1}\left(\mathbb{R}^{m}\right)$. For binary schemes

$$
\left\{\begin{array}{l}
(S f)_{2 n}=\psi_{0}\left(f_{n-p}, \ldots, f_{n+p}\right) \\
(S f)_{2 n+1}=\psi_{1}\left(f_{n-p}, \ldots, f_{n+p}\right)
\end{array}\right.
$$

such that $\psi_{k} \in C_{p w}^{1}\left(\mathbb{R}^{2 p+1}\right), k=0,1$, a Generalized Jacobian of $S$ at $f \in l_{\infty}(\mathbb{Z})$, $D S(f)$, is defined as the linear operator associated to the bi-infinite matrix whose rows have the following non-zero components

$$
(D S(f))_{[2 n+k, n-p: n+p]}=D \psi_{k}\left(f_{n-p}, \ldots, f_{n+p}\right), \quad k=0,1, \quad j \in \mathbb{Z},
$$

where $D \psi_{k}$ is a generalized gradient of the function $\psi_{k}$. We notice that $\forall f, g \in$ $l_{\infty}(\mathbb{Z}), k=0,1$

$\left|(D S(f) g)_{2 n+k}\right|=\left|D \psi_{k}\left(f_{n-p}, \ldots, f_{n+p}\right) \cdot\left(g_{n-p}, \ldots, g_{n+p}\right)\right| \leq\left\|D \psi_{k}\left(f_{n-p}, \ldots, f_{n+p}\right)\right\|_{1}\|g\|_{\infty}$ hence

$$
\|D S(f)\|_{\infty}=\sup _{g \neq 0} \frac{\|D S(f) g\|_{\infty}}{\|g\|_{\infty}} \leq \max \left\{C_{1}^{\psi_{0}}, C_{1}^{\psi_{1}}\right\}
$$

with $C_{1}^{\psi_{k}}$ in (A.1). The following result generalizes Theorem 22.

Theorem 23 Let $S$ be a scheme of the form (A.16), $\psi_{k} \in C_{p w}^{1}\left(\mathbb{R}^{2 p+1}\right)$, and let $\gamma:[a, b] \rightarrow l_{\infty}(\mathbb{Z})$ be a Lipschitz curve ${ }^{4}$. Then $\tilde{\gamma}=S \circ \gamma:[a, b] \rightarrow l_{\infty}(\mathbb{Z})$ is also a Lipschitz curve, and

$$
\tilde{\gamma}^{\prime}(t)=D S(\gamma(t)) \gamma^{\prime}(t) \quad \text { a.e. on }(a, b) .
$$

Proof In each coordinate we have

$$
\tilde{\gamma}_{2 j+k}=(S \gamma)_{2 j+k}=\psi_{k} \circ \chi_{j-p, j+p} \circ \gamma,
$$

with $\chi_{n, m} f=\left(f_{n}, \ldots, f_{m}\right), n<m$. Since $\psi_{k}$ is Lipschitz, we have that

$$
\begin{aligned}
\left\|\tilde{\gamma}_{2 j+k}(s)-\tilde{\gamma}_{2 j+k}(t)\right\|_{\infty} & \leq L_{\psi_{k}}\left\|\left(\chi_{j-p, j+p} \circ \gamma\right)(s)-\left(\chi_{j-p, j+p} \circ \gamma\right)(t)\right\|_{\infty} \\
& \leq L_{\psi_{k}}\|\gamma(s)-\gamma(t)\|_{\infty} \leq L_{\psi_{k}} L_{\gamma}|s-t|,
\end{aligned}
$$

${ }^{4} \gamma=\left\{\gamma_{i}\right\}_{i \in \mathbb{Z}}$ with $\gamma_{i}: \mathbb{R} \rightarrow \mathbb{R}$ and $\left|\gamma_{i}(x)-\gamma_{i}(y)\right| \leq L_{\gamma}|x-y|, \forall x, y \in \mathbb{R}, \forall i \in \mathbb{Z}$. 
where $L_{*}$ denotes the Lipschitz constant of each function involved. Therefore, each component of $\tilde{\gamma}$ is a Lipschitz curve and

$$
\|\tilde{\gamma}(s)-\tilde{\gamma}(t)\|_{\infty}=\sup _{i \in \mathbb{Z}}\left|\tilde{\gamma}_{i}(s)-\tilde{\gamma}_{i}(t)\right| \leq \max \left\{L_{\psi_{0}}, L_{\psi_{1}}\right\} L_{\gamma}|s-t| .
$$

Since the countable union of sets of zero measure has zero measure, Eq. A.18 follows as in Theorem 22.

Obviously, the last two theorems apply as long as the functions $\psi_{i}$ admit uniformly bounded Generalized Gradients, $D \psi_{i}$, satisfying the chain rule (A.10).

\section{The study of contractivity via Generalized Jacobians}

Theorem 23 allows to study the contractivity properties of the powers of subdivision schemes that are defined by functions in $C_{p w}^{1}\left(\mathbb{R}^{m}\right)$ (or, in general, by functions that admit uniformly bounded Generalized Gradients satisfying the chain rule (A.10)) by the following argument, sketched in [19]:

Given $f, g \in l_{\infty}(\mathbb{Z})$, define recursively $\gamma^{j}:[0,1] \rightarrow l_{\infty}(\mathbb{Z})$ as follows

$$
\gamma^{0}(t):=t f+(1-t) g, \quad \gamma^{j}(t):=S \circ \gamma^{j-1}(t), \quad j>0 .
$$

Notice that $\left(\gamma^{0}\right)^{\prime}(t)=f-g$, hence $\gamma^{j}$ is also Lipschitz and

$$
\begin{aligned}
\left(\gamma^{j}\right)^{\prime}(t) & =D S\left(\gamma^{j-1}(t)\right)\left(\gamma^{j-1}(t)\right)^{\prime}=\ldots \\
& =D S\left(\gamma^{j-1}(t)\right) D S\left(\gamma^{j-2}\right)(t) \cdots D S\left(\gamma^{0}(t)\right)\left(\gamma^{0}\right)^{\prime}(t), \quad \text { a.e. in }(0,1) .
\end{aligned}
$$

Hence, since $\gamma^{j}(1)=S^{j} f, \gamma^{j}(0)=S^{j} g, \forall j \geq 0$ we can write

$$
\begin{gathered}
S^{j} f-S^{j} g=\gamma^{j}(1)-\gamma^{j}(0)=\int_{0}^{1} D S\left(\gamma^{j-1}(t)\right) D S\left(\gamma^{j-2}\right)(t) \cdots D S\left(\gamma^{0}(t)\right)(f-g) d t, \\
\left\|S^{j} f-S^{j} g\right\|_{\infty} \leq\left(\int_{0}^{1}\left\|\Pi_{k=0}^{j-1} D S\left(\gamma^{k}(t)\right)\right\|_{\infty} d t\right)\|f-g\|_{\infty} .
\end{gathered}
$$

From Eq. A.20, we easily deduce the following contractivity result, which has been used in [22] to prove the stability of a monotone nonlinear scheme.

Corollary 24 Let us assume that $\exists L \geq 1,0<\mu<1$ s. t. $\forall f_{0}, \ldots, f_{L} \in l_{\infty}(\mathbb{Z})$, $\left\|\Pi_{k=0}^{L-1} D S\left(f_{k}\right)\right\|_{\infty} \leq \mu$, then $S^{L}$ is contractive.

\section{References}

1. Amat, S., Dadourian, K., Liandrat, J.: Analysis of a class of nonlinear subdivision schemes and associated multiresolution transforms. Adv. Comput. Math. 34(3), 253-277 (2011)

2. Amat, S., Donat, R., Liandrat, J., Trillo, J.C.: Analysis of a new nonlinear subdivision scheme. Applications in image processing, Applications in Image Processing. Found Comput. Math., 193-225 (2006)

3. Dadourian, K., Liandrat, J.: Analysis of some bivariate non-linear interpolatory subdivision schemes. Numer. Algorithm. 48, 261-278 (2008) 
4. Amat, S., Donat, R., Liandrat, J., Trillo, J.C.: A fully adaptive multiresolution scheme for image processing. Math. Comput. Modell. 46, 2-11 (2007)

5. Kuijt, F.: Convexity preserving interpolation. Stationary nonlinear subdivision and splines. $\mathrm{PhD}$ thesis, University of Twente, The Netherlands (1998)

6. Deslauriers, G., Dubuc, S.: Interpolation dyadique: fractals, dimension non entieres et application. Masson Paris, 44-45 (1987)

7. Clarke, F.H., Ledyae, Y.S.: Mean value inequalities. Proc. Am. Math. Soc. 122, 1075-1083 (1994)

8. Kuijt, F., Van Damme, R.: Monotonicity preserving interpolatory subdivision scheme. J. Comput. Appl. Math. 101, 203-229 (1999)

9. Aràndiga, F., Donat, R., Harten, A.: Multiresolution based on weighted averages of the hat function i: linear reconstruction techniques. SIAM J. Numer. Anal. 36, 160-203 (1999)

10. Harten, A.: Multiresolution representation of data: a general framework. SIAM J. Numer. Anal. 33, 1205-1256 (1996)

11. Aràndiga, F., Donat, R.: Nonlinear multi-scale decomposition: the approach of A. harten. Numer. Algorithm. 23, 175-216 (2000)

12. Daubechies, I., Runborg, O., Sweldens, W.: Normal multiresolution approximation of curves. Constr. Approx. 20, 399-463 (2003)

13. Burden, R.L., Douglas Faires, J.: Numerical analysis. Thomson Brooks/Cole (2005)

14. Aràndiga, F., Belda, A.M., Mulet, P.: Point-value WENO multiresolution applications to stable image compression. J. Sci. Comput. 43, 158-182 (2010)

15. Marquina, A., Serna, S.: Power ENO methods: a fifth-order accurate weighted power ENO method. J. Comput. Phys. 194(2), 632-658 (2004)

16. Cohen, A., Dyn, N., Matei, B.: Quasilinear subdivision schemes with applications to ENO interpolation. Appl. Comput. Harmon. Anal. 15, 89-116 (2003)

17. Dadourian, K.: Schémas de Subdivision, Analyses Multirésolutions non-linéaires, Applications, PhD thesis, Université de Provence (2008)

18. Xie, G., Yu, T.P.-Y.: Smoothness analysis of nonlinear subdivision schemes of homogeneous and affine invariant type. Constr. Approx. 22, 219-254 (2005)

19. Harizanov, S., Oswald, P.: Stability of nonlinear subdivision and multiscale transforms. Constr. Approx. 31(3), 359-393 (2010)

20. Dyn, N.: Subdivision schemes in computer-aided geometric design, Advances in numerical analysis, Vol. II (Lancaster, 1990), pp. 36-104. Oxford Science of Publication, Oxford University Press, New York (1992)

21. Imbert, C.: Support functions of the Clarke generalized Jacobian and of its plenary hull. Nonlinear Anal. 49, 1111-1125 (2002)

22. Aràndiga, F., Donat, R., Santágueda, M.: The PCHIP subdivision scheme. J. Comput. Appl. Math. 272, 28-40 (2016)

23. Aràndiga, F., Donat, R., Santágueda, M.: Weighted-Power $p$ nonlinear subdivision schemes. Curv. Surf. 6920, 109-129 (2012) 